\title{
nawdex
}

NATIONAL WATER DATA EXCHANGE

\section{DIRECTORY OF MEMBER ORGANIZATIONS OF THE NATIONAL WATER DATA EXCHANGE (NAWDEX)}

U.S. GEOLOGICAL SURVEY

Open-File Report 90-141 


\section{DIRECTORY OF MEMBER ORGANIZATIONS OF THE NATIONAL WATER DATA EXCHANGE (NAWDEX)}

By Cassandra D. Blackwell

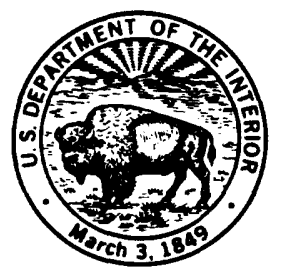

U.S. GEOLOGICAL SURVEY

Open-File Report 90-141

(Supersedes Open-File Report 89-44) 


\section{DEPARTMENT OF THE INTERIOR MANUEL LUJAN, JR., Secretary U.S. GEOLOGICAL SURVEY Dallas L. Peck, Director}

For additional information write to:

Chief, Hydrologist U.S. Geological Survey 421 National Center Reston, Virginia 22092
Copies of this report can be purchased from:

U.S. Geological Survey Books and Open-File Reports Section Box 25425, Federal Center Denver, Colorado 80225 
Abstract . . . . . . . . . . . . . . . . . . . . . . . . . . . 1

Introduction . . . . . . . . . . . . . . . . . . . . . . . . . 1

National Water Data Exchange membership . . . . . . . . . . . . . . . . 1

Purpose of the directory . . . . . . . . . . . . . . . . . . . . 2

Additional information. . . . . . . . . . . . . . . . . . . . 2

Directory of member organizations . . . . . . . . . . . . . . . . . . . 3

Acheron Engineering Services . . . . . . . . . . . . . . . . . . . 3

Agricultural Research Service (USDA). . . . . . . . . . . . . . 3

Alabama Geological Survey . . . . . . . . . . . . . . . . . . . . 3

Alameda County, Office of Education . . . . . . . . . . . . . . . 3

Alaska Department of Environmental Conservation . . . . . . . . . 3

Alaska Department of Fish and Game--Habitat Division . . . . . . . 4

Alaska Department of Natural Resources . . . . . . . . . . . . . . 4

Alaska Department of Transportation and Public Facilities . . . . 4

Albert Mann Library - Cornell University . . . . . . . . . . . . . 4

Aqua Resources, Inc. . . . . . . . . . . . . . . . . . . . . . 4

Aqua Terra Consultants . . . . . . . . . . . . . . . . . . 5

Aquatic Systems Branch . . . . . . . . . . . . . . . . . . . . 5

Arco Oil and Gas Company . . . . . . . . . . . . . . . . . . . . . 5

Arkansas Department of Pollution Control and Ecology . . . . . . . 5

Arkansas Soil and Water Conservation Commission. . . . . . . . . . 5

Army Corps of Engineers . . . . . . . . . . . . . . . . . . . . 6

Ayres Associates. . . . . . . . . . . . . . . . . . . . 6

BCA Environmental . . . . . . . . . . . . . . . . . 6

BCI Geonetics, Inc. . . . . . . . . . . . . . . . . . . 6

BCM Eastern, Inc. . . . . . . . . . . . . . . . . . . . . . 7

Bechtel Corporation . . . . . . . . . . . . . . . . . . . . . 7

Besha Associates Engineering Corporation . . . . . . . . . . . . . 7

Bhate Engineering Corporation . . . . . . . . . . . . . . . . . . 7

Bingham Engineering . . . . . . . . . . . . . . . . . . . . . . . 7

Black and Veatch Engineers-Architects . . . . . . . . . . . . . . 8

Bonneville Power Administration (USDOE) . . . . . . . . . . . . . . . . 8

Bookman-Edmonston Engineering, Inc. . . . . . . . . . . . . . . . 8

Boyle Engineering Corporation . . . . . . . . . . . . . . . . . 8

Bromwell and Carrier, Inc. . . . . . . . . . . . . . . . . . . . . 8

Brookhaven National Laboratory . . . . . . . . . . . . . . . . . 9

Bureau of Indian Affairs (U.S.) . . . . . . . . . . . . . . . . . 9

Bureau of Land Management (U.S.) . . . . . . . . . . . . . . . . . 9

Bureau of Reclamation (U.S.) . . . . . . . . . . . . . . . . . . . 11

Burns and McDonnell .. . . . . . . . . . . . . . . . . . . . . . 11 
CH2M Hill . . . . . . . . . . . . . . . . . . . . . . . 11

Calexco, Inc. . . . . . . . . . . . . . . . . . . . . 12

Calgon Corporation . . . . . . . . . . . . . . . . . . . . . 12

California Department of Water Resources . . . . . . . . . . . . . 12

Calpine Corporation . . . . . . . . . . . . . . . . . . . . . 12

Carolina Power \& Light Company . . . . . . . . . . . . . . . . . . 12

Carr/Associates . . . . . . . . . . . . . . . . . . . . . . . . 13

C-b Site, Occidental Oil Shale, Inc. . . . . . . . . . . . . . . 13

Center for Water Resources Research, Utah State University . . . . 13

Chas T. Main, Inc. . . . . . . . . . . . . . . . . . . . . . . 13

Chen-Northern, Inc. . . . . . . . . . . . . . . . . . . . . . 13

Christensen Development Corporation . . . . . . . . . . . . . . 14

City of Aurora, Utilities Administration . . . . . . . . . . . . . 14

City of Everett Engineering Department . . . . . . . . . . . . . . 14

City of Savannah Industrial and Domestic Water Supply . . . . . . 14

Clement Associates... . . . . . . . . . . . . . . . . . . . 14

Colorado Division of Water Resources . . . . . . . . . . . . . . . 15

Colorado River Water Conservation District. . . . . . . . . . . . 15

Colorado State University, Department of Agricultural and

Chemical Engineering. . . . . . . . . . . . . . . . . . . 15

Computer Innovations . . . . . . . . . . . . . . . . . . . . . . 15

Consolidated Hydro, Inc. . . . . . . . . . . . . . . . . . . 15

Council on Environmental Quality (U.S.)............. . 16

Department of Civil Engineering . . . . . . . . . . . . . . . . . 16

Department of Energy (U.S.) . . . . . . . . . . . . . . . . . . 16

Department of Geology, San Diego State University . . . . . . . . . 16

Deuel and Associates, Inc. . . . . . . . . . . . . . . . 16

Dewberry and Davis . . . . . . . . . . . . . . . . . . . . . 17

Dow Chemical, U.S.A. - Earth Sciences. . . . . . . . . . . . . 17

Duke University, School of Engineering . . . . . . . . . . . . . . 17

Dunn Geoscience Corporation . . . . . . . . . . . . . . . . . . . 17

Dynamac Corporation . . . . . . . . . . . . . . . . . . . . . . 17

E \& S Environmental Chemistry . . . . . . . . . . . . . . . . . . . 18

Ebasco Environmental . . . . . . . . . . . . . . . . . 18

Ebasco Services Incorporated . . . . . . . . . . . . . . . . . . 18

Edward E. Clark Engineers-Scientists, Inc. . . . . . . . . . . . . 18

Electric Power Research Institute . . . . . . . . . . . . . . . . 18

Engineering Methods and Applications, Inc. . . . . . . . . . . . . 19

Environmental Biology Research Program . . . . . . . . . . . . . . 19

Environmental Defense Fund . . . . . . . . . . . . . . . . . . . . 19

Environmental Protection Agency (U.S.) . . . . . . . . . . . . . . 19

Environmental Protection Agency, Regions III, IV, V, VII and X . . 20

Environmental Research and Technology, Inc. . . . . . . . . . . . . 21

Environmental Resources Management, Inc. . . . . . . . . . . . . . 21

Espey, Huston \& Associates, Inc. . . . . . . . . . . . . . . . . . 21

FMC Corporation . . . . . . . . . . . . . . . . . . . . . . . . 21

Federal Energy Regulatory Commission (U.S.) . . . . . . . . . . . 21

Federal Highway Administration . . . . . . . . . . . . . . . . . 22

Fish and Wildlife Service (U.S.) . . . . . . . . . . . . . . . . 22

Florida Department of Environmental Regulation . . . . . . . . . . 22

Florida Institute of Phosphate Research . . . . . . . . . . . . . . 22 
Forest Service (U.S.) . . . . . . . . . . . . . 23

includes:

Directors of Soil, Watershed, and Air Management . . . . . . . 23

Forest Service Research Stations... . . . . . . . . . . . . 24

State and Private Forestry Areas . . . . . . . . . . . . . . . 24

Fort Dodge Department of Municipal Utilities . . . . . . . . . . . 25

GAI Consultants... . . . . . . . . . . . . . . . . . . . 25

GANCOM, Inc. . . . . . . . . . . . . . . . . . . . . . . . . . 25

Geauga County Office of the Sanitary Engineer, (Ohio) . . . . . . . 25

General Sciences Corporation . . . . . . . . . . . . . . . 25

Geological Survey (U.S.) . . . . . . . . . . . . . . . . 26

GeoResearch, Inc. . . . . . . . . . . . . . . . . . . . 31

Geraghty and Miller, Inc. . . . . . . . . . . . . . . . . . . . 31

Gidley Laboratories, Inc. . . . . . . . . . . . . . . . . . . . . . 31

Gill and Pulver Engineers, Inc. . . . . . . . . . . . . . . . . . 31

Greenhorne and $0^{\prime}$ Mara, Inc. . . . . . . . . . . . . . . . . . . . . 31

Greiner Engineering . . . . . . . . . . . . . . . . . . . . . . 32

HRS Water Consultants, Inc. . . . . . . . . . . . . . . . . . . 32

Handar, Inc. . . . . . . . . . . . . . . . . . . . . . . 32

Harmsworth Associates . . . . . . . . . . . . . . . . . . . . . 32

Hazen and Sawyer, P. C. . . . . . . . . . . . . . . . . . . . 32

Herbert E. Skibitzke, P.E. . . . . . . . . . . . . . . . . . . . 33

Hetch Hetchy Water and Power . . . . . . . . . . . . . . . . . . 33

Hittman Ebasco Associates, Inc. . . . . . . . . . . . . . . . . . . 33

Honolulu Board of Water Supply . . . . . . . . . . . . . . . . . . 33

Hydro Research Science .. . . . . . . . . . . . . . . . . . 33

Hydro-Triad, LTD . . . . . . . . . . . . . . . . . . . 34

Hydromet Data Services, Inc. . . . . . . . . . . . . . . . . . . . 34

Illinois Department of Transportation, Division of Water
Resources.... . . . . . . . . . . . . . . . 34

Illinois State Water Survey . . . . . . . . . . . . . . . . 34

In-Situ, Inc. . . . . . . . . . . . . . . . . . . . . . . . . . 34

International Boundary and Water Commission . . . . . . . . . . . . 35

International Ground Water Modeling Center (IGWMC) . . . . . . . . 35

Interstate Commission on the Potomac River Basin . . . . . . . . . 35

Iowa Department of Natural Resources, Geological Survey Bureau . . 35

Iowa Institute of Hydraulic Research, University of Iowa . . . . . 35

JBS Engineering, CA . . . . . . . . . . . . . . . . . . . 36

J. Venn Leeds, Jr. . . . . . . . . . . . . . . . . . . . 36

J. R. Nuckols, Ph.D., C.P.H. . . . . . . . . . . . . . . . . 36

J. W. Patterson and Associates, Inc. . . . . . . . . . . . . . 36

James M. Montgomery Consulting Engineers, Inc. . . . . . . . . . . 36

John C. Landon and Associates, Inc. . . . . . . . . . . . . . . . 37

Johnson and Anderson, Inc. . . . . . . . . . . . . . . . . . . . . 37

Joint Montana University, Water Research Center . . . . . . . . . . 37

Jones and Stokes Associates. . . . . . . . . . . . . . . . . . . 37

K. M. $\mathrm{Ng}$ and Associates, Inc. . . . . . . . . . . . . . . . . . 37

Kansas State Board of Agriculture . . . . . . . . . . . . . . . . . 38

Kearney Waste Water Treatment Plant. . . . . . . . . . . . . . . . 38

Kentucky Department of Environmental Protection . . . . . . . . . . 38

Kindle, Stone and Associates, Inc. . . . . . . . . . . . . . . . 38 
King County Department of Public Works . . . . . . . . . . . . . . 38

Lake County Water Authority . . . . . . . . . . . . . . . . . . . 39

Lake Mary Water Department . . . . . . . . . . . . . . . . . . . 39

Law Environmental, Inc. . . . . . . . . . . . . . . . . . . . . 39

Lawrence Berkeley Laboratory . . . . . . . . . . . . . . . . . . . 39

Lawrence Livermore Laboratory . . . . . . . . . . . . . . . . . . . 39

Leonard Rice Consulting Water Engineers, Inc. . . . . . . . . . . . 40

Los Angeles County Department of Public Works . . . . . . . . . . . 40

Louisville Water Company . . . . . . . . . . . . . . . . . . . . . 40

Maine Geological Survey .. . . . . . . . . . . . . . . . . 40

Marin Municipal Water District . . . . . . . . . . . . . . . 40

Marine Resources Council . . . . . . . . . . . . . . . . . . . . . 41

Maryland Natural Resources, Water Resources Administration . . . . 41

McCall-Ellingson and Morrill, Inc. . . . . . . . . . . . . . . . . 41

McLaren Environmental Engineering . . . . . . . . . . . . . . . . . 41

Merced Irrigation District . . . . . . . . . . . . . . . . . . . . 41

Metropolitan Denver Sewage Disposal District No. 1 . . . . . . . . 42

Metropolitan District Water Bureau . . . . . . . . . . . . . . . . 42

Millipore Corporation . . . . . . . . . . . . . . . . . . . . . 42

Minnesota Dept. of Health, Div. of Environmental Health . . . . . . 42

Minnesota Pollution Control Agency . . . . . . . . . . . . . . . . 42

Mississippi Bureau of Geology, Dept. of Natural Resources . . . . . 43

Mississippi Bureau of Land and Water Resources . . . . . . . . . . 43

Mississippi State University . . . . . . . . . . . . . . . . . . . 43

Missouri Division of Geology and Land Survey . . . . . . . . . . . 43

Monroe County (NY) Water Authority . . . . . . . . . . . . . . . . 43

Monsanto Agricultural Company . . . . . . . . . . . . . . . . . . . 44

Montana Bureau of Mines and Geology . . . . . . . . . . . . . . . . 44

Montana Department of Natural Resources and Conservation . . . . . 44

Montgomery County Department of Environmental Protection . . . . . 44

Morrison-Knudsen Engineers, Inc. . . . . . . . . . . . . . . . . . 44

Mountain Valley Water . . . . . . . . . . . . . . . . . . . 45

Multitech, Inc. . . . . . . . . . . . . . . . . . . . . . 45

N.U.S. Corporation . . . . . . . . . . . . . . . . . . . . . . 45

National Ecology Research Center - Leetown . . . . . . . . . . . . 45

National Fisheries Contaminant Research Center, U.S. Fish and Wildlife Services . . . . . . . . . . . . . . . . . 45

National Oceanic and Atmospheric Administration . . . . . . . . . . 46 including:

National Weather Service. . . . . . . . . . . . . . . 46

National Ocean Service. . . . . . . . . . . . . . . . . . . 46

National Marine Fisheries Service . . . . . . . . . . . . . . 46

Environmental Research Laboratories . . . . . . . . . . . . . 46

Great Lakes Environmental Research Laboratory . . . . . . . . 46

National Environmental Satellite, Data and Information Service includes following data centers:

The National Climatic Center (NCDC) . . . . . . . . . . . . 46

The National Oceanographic Data Center (NODC) . . . . . . . 46

Climate Applications Branch . . . . . . . . . . . . . . . . 47 
National Ground Water Information Center . . . . . . . . . . . . . 47

National Park Service, Everglades National Park . . . . . . . . . . 47

Natural Science Systems . . . . . . . . . . . . . . . . . . . . . . 48

Nebraska Natural Resources Commission . . . . . . . . . . . . . . . 48

Nevada Department of Conservation and Natural Resources . . . . . . 48

New Castle County Water Resources Agency . . . . . . . . . . . . . 48

New Jersey Geological Survey . . . . . . . . . . . . . . . . . . . 48

New Mexico Environmental Improvement Division . . . . . . . . . . . 49

New Mexico State Engineers Office... . . . . . . . . . . . . . . 49

New York State Department of Environmental Conservation . . . . . . 49

Noah Corporation . . . . . . . . . . . . . . . . . . . . 49

North American Hydro, Inc. . . . . . . . . . . . . . . . . . . . . 49

North Carolina Department of Natural Resources and

Community Development . . . . . . . . . . . . . . . . . 50

North Dakota Geological Survey . . . . . . . . . . . . . . . . . . 50

North Dakota State Water Commission. . . . . . . . . . . . . . . . 50

Northeast Engineering Associates, Inc. . . . . . . . . . . . . . 50

Northwest Florida Water Management District . . . . . . . . . . . . 50

Northwest Hydraulic Consultants, Inc. . . . . . . . . . . . . . . 51

Nuclear Regulatory Commission (U.S.) . . . . . . . . . . . . . 51

OTT Engineering, Inc. . . . . . . . . . . . . . . . . . . . . . . 51

Oak Ridge National Laboratory . . . . . . . . . . . . . . . . . . . 51

Office of Surface Mining Reclamation and Enforcement . . . . . . . 52

Ohio Environmental Protection Agency . . . . . . . . . . . . . . . 52

Ohio River Valley Water Sanitation Commission . . . . . . . . . . . 52

Oklahoma Climatological Survey . . . . . . . . . . . . . . . . 52

Oklahoma State Department of Health . . . . . . . . . . . . . . . . 53

Oklahoma State University, Department of Geology . . . . . . . . . 53

Oklahoma Water Resources Board . . . . . . . . . . . . . . . . . 53

P. E. LaMoreaux and Associates . . . . . . . . . . . . . . . . . . 53

P. G. International, Inc. . . . . . . . . . . . . . . . . . . 53

PTI Environmental Services . . . . . . . . . . . . . . . . . . . . 54

Parsons, Brinckerhoff, Quade and Douglas, Inc. . . . . . . . . . 54

Pat Harrison Waterway District . . . . . . . . . . . . . . . . . . 54

Pendletın, Oregon, Public Works . . . . . . . . . . . . . . . . . . 54

Pennsyl znia Department of Environmental Resources . . . . . . . . 54

Pollution Engineering . . . . . . . . . . . . . . . . . . . . . 55

Post, Buckley, Schuh, and Jernigan, Inc. . . . . . . . . . . . . . 55

RMC-Environmental Services . . . . . . . . . . . . . . . . . 55

R. E. Wright Associates, Inc. . . . . . . . . . . . . . . . . . . 55

R. W. Beck and Associates . . . . . . . . . . . . . . . . . . . . 55

$\mathrm{R}$ and $\mathrm{M}$ Consultants, Inc. . . . . . . . . . . . . . . . . . . . 56

Radian Environmental Management . . . . . . . . . . . . . . . . 56

Randolph and Associates, Inc . . . . . . . . . . . . . . . . . 56

Raymond Vail and Associates . . . . . . . . . . . . . . . . 56

Reedy Creek Improvement District . . . . . . . . . . . . . . 56

Research Triangle Institute . . . . . . . . . . . . . . . . . . . . 57

Rio Blanco Oil Shale Co. . . . . . . . . . . . . . . . . . . 57

Roald Haestad, Inc. . . . . . . . . . . . . . . . . . . . . . . . 57

Rocky Mountain Hydro, Inc . . . . . . . . . . . . . . . . . 57 
Roy F. Weston, Inc., Weston Environmental

Consultants-Designers . . . . . . . . . . . . . . . . . 57

SCS Engineers... . . . . . . . . . . . . . . . . . . . . . 58

SS Papadopulos and Associates . . . . . . . . . . . . . . . . . . 58

Salt River Project . . . . . . . . . . . . . . . . . . . . . 58

Santa Clara (City of) Water Department . . . . . . . . . . . . . . 58

Santa Clara Valley Water District, Calif. . . . . . . . . . . . . . 58

Sargent \& Lundy Engineers . . . . . . . . . . . . . . . . . . . . . 59

Scripps Institution of Oceanography . . . . . . . . . . . . . . . . 59

Sierra/Misco, Inc. . . . . . . . . . . . . . . . . . . . . . . . 59

Simons, Li and Associates, Inc. . . . . . . . . . . . . . . . 59

Sithe Energies USA, Inc. . . . . . . . . . . . . . . . . . . . . 59

Soil Conservation Service. . . . . . . . . . . . . . . . . . 60

Snow Survey and Water Supply Forecasting . . . . . . . . . . 60

Vermont SCS District Office . . . . . . . . . . . . . . . . . . 60

Sonoma County Water Agency . . . . . . . . . . . . . . . . . . . . 60

South Florida Environmental Research Foundation. . . . . . . . . . 60

Southern California Edison Company . . . . . . . . . . . . . . . . 61

Southern Company Services, Inc. . . . . . . . . . . . . . . . . . . 61

Southwest Florida Water Management District . . . . . . . . . . . . 61

Southwest Georgia Planning and Development Commission . . . . . . . 61

State of South Carolina, Water Resources Commission . . . . . . . . 61

Stetson Engineers, Inc. . . . . . . . . . . . . . . . . . . . . . . 62

Susquehanna River Basin Commission . . . . . . . . . . . . . . . . 62

Suwannee River Water Management District . . . . . . . . . . . . . 62

Systems for Water Information Management . . . . . . . . . . . . . 62

Tampa Bay Engineering, Inc. . . . . . . . . . . . . . . . . . . . . 62

Tenera, L. P. . . . . . . . . . . . . . . . . . . . . . . 63

TenEch Environmental Engineers, Inc. . . . . . . . . . . . . . 63

Tennessee Department of Health and Environment . . . . . . . . . . 63

Tennessee Valley Authority . . . . . . . . . . . . . . . . 63

Tetra Tech, Inc. . . . . . . . . . . . . . . . . . . . . . . 64

Texas Natural Resources Information System . . . . . . . . . . . 64 representing:

Texas Water Development Board . . . . . . . . . . . . . . . . . 64

Texas General Land Office. . . . . . . . . . . . . . . . . . . 64

Texas Air Control Board. . . . . . . . . . . . . . . . . . . . 64

Texas Forest Service . . . . . . . . . . . . . . . . . . . . 65

Texas Economic Development Commission . . . . . . . . . . . . . . 65

Texas Department of Health . . . . . . . . . . . . . . . . . . 65

Bureau of Economic Geology, (University of Texas at Austin) . . . 65

Railroad Commission of Texas . . . . . . . . . . . . . . . . . 65

Texas Department of Agriculture . . . . . . . . . . . . . . . . . 65

Texas Historical Commission . . . . . . . . . . . . . . . . . . . 66

Texas State Department of Highways and Public Transportation . . 66

Texas State Soil and Water Conservation Board . . . . . . . . . . 66

Texas Water Commission . . . . . . . . . . . . . . . . . . . . 66

The Chester Engineers, Inc. . . . . . . . . . . . . . . . . . . 66

The Dow Chemical Company . . . . . . . . . . . . . . . . . . 67

The Environ Corporation... . . . . . . . . . . . . . . . 67 
Tudor Engineering Company . . . . . . . . . . . . . . . . . 67

Uintex Corporation . . . . . . . . . . . . . . . . . . . . . . . . 67

University of Arizona, College of Business and Public

Administration . . . . . . . . . . . . . . . . . . . . . . . 67

University of California at Santa Cruz . . . . . . . . . . . . . . 68

University of Maryland, Dept of Civil Engineering . . . . . . . . . 68

Updata Publications, Inc. . . . . . . . . . . . . . . . . . . 68

Upper Colorado River Commission . . . . . . . . . . . . . . . . . 68

Utah Department of Natural Resources, Division of Water Rights . 68

Vermont Department of Environmental Conservation . . . . . . . . . 69

Versar, Inc. . . . . . . . . . . . . . . . . . . . . . . 69

Virginia Beach Department of Public Utilities . . . . . . . . . . . 69

WBLA, Inc. . . . . . . . . . . . . . . . . . . . . . . 69

W. W. Wheeler and Associates . . . . . . . . . . . . . . 69

Wagner, Heindel, and Noyes, Inc. . . . . . . . . . . . . . . 70

Wapora, Inc. . . . . . . . . . . . . . . . . . . . . 70

Warzyn Engineering, Inc. . . . . . . . . . . . . . . . . . . . 70

Washington Department of Ecology . . . . . . . . . . . . . . . . 70

Washington Department of Fisheries, Habitat Management Division . . 70

Water and Energy Research Institute, University of Guam . . . . . . 71

Water Engineering and Technology, Inc. . . . . . . . . . . . . . . 71

Water Research Center, Washington State University . . . . . . . . 71

Water Resources Center:

Nevada University System . . . . . . . . . . . . . . 71

Ohio State University . . . . . . . . . . . . . . . 71

Rhode Island University . . . . . . . . . . . . . . . 72

University of Delaware . . . . . . . . . . . . . . . . . 72

University of Illinois at Urbana-Champaign . . . . . . . . . . 72

University of Wisconsin . . . . . . . . . . . . . . . . . . 72

Water Resources Research Center:

Arizona University . . . . . . . . . . . . . . . . . 72

District of Columbia University . . . . . . . . . . . . 73

Massachusetts University . . . . . . . . . . . . . 73

University of Minnesota . . . . . . . . . . . . . . 73

University of Missouri . . . . . . . . . . . . . . . . . . 73

University of New Hampshire . . . . . . . . . . . . . . . . 73

Virginia Polytechnic Institute and State University . . . . . 74

Water Resources Research Institute:

North Dakota State University . . . . . . . . . . . . . . 74

South Dakota State University . . . . . . . . . . . . . . . 74

University of Idaho .. . . . . . . . . . . . . . . . . . . . 74

University of Kentucky . . . . . . . . . . . . . . . . . . . . 74

University of Puerto Rico . . . . . . . . . . . . . . . 75

University of North Carolina . . . . . . . . . . . . . . 75

WaterTest Corporation... . . . . . . . . . . . . . . . . 75

Western Engineers, Inc. . . . . . . . . . . . . . . . . . . . 75

Western Hydrologic Systems . . . . . . . . . . . . . . . . . 75

William T. Lorenz and Company . . . . . . . . . . . . . . . . . . 76

Winter Haven Lake Region Boat Course District . . . . . . . . . . . 76

Wisconsin Geological and Natural History Survey . . . . . . . . . . 76 
Woodward-Clyde Consultants . . . . . . . . . . . . . 76

Wright-Pierce Engineers . . . . . . . . . . . . . . . . . . . 76

Wright Water Engineers, Inc. . . . . . . . . . . . . . . . . . . . 77

Wyoming Department of Environmental Quality . . . . . . . . . . . . 77

Wyoming Water Research Center, University of Wyoming . . . . . . . 77

Yonts and Associates . . . . . . . . . . . . . . . . . . . . . . 77

Young Environmental Services, Inc. . . . . . . . . . . . . . . . . 77

Foreign Affiliates:

Aqua Mex., S.A., Mexico . . . . . . . . . . . . . . . . . . . 78

Companhia Auxiliar de Empresas Electricas Brasileiras (CAEEB) . . . . . . . . . . . . . . . . . . . . 78

National Institute of Hydrology . . . . . . . . . . . . . . 78

Nigerian Water Data Bank . . . . . . . . . . . . . . . . . 78

South Africa Water Information Centre, CSIR . . . . . . . . . 79

Water Resources Document Reference Centre, Inland

Waters Directorate, Canadian Department of

the Environmental . . . . . . . . . . . . . . . . . . . 79

Water Resources Planning Commission . . . . . . . . . . . 79

Appendix . . . . . . . . . . . . . . . . . . . . . . . . . 80 


\author{
DIRECTORY OF MEMBER ORGANIZATIONS \\ OF THE \\ NATIONAL WATER DATA EXCHANGE (NAWDEX)
}

By

Cassandra D. Blackwell

\begin{abstract}
The National Water Data Exchanage (NAWDEX) is a national confederation of water-oriented organizations working together to improve access to water data. It consists of member organizations from all sectors of the water-data community. This Directory provides the names, addresses, and telephone numbers of all NAWDEX member organizations and their designated NAWDEX representatives.
\end{abstract}

\title{
INTRODUCTION
}

The National Water Data Exchange (NAWDEX) is a national program to assist users of water data or water-related data in identifying, locating, and acquiring needed data. A variety of data, indexing, assistance and referral services are provided through a nationwide network of Assistance Centers located in 45 states and Puerto Rico. The program is centrally managed and coordinated by a NAWDEX Program office located within the U.S. Geological Survey in Reston, Va.

NAWDEX is a national confederation consisting of member organizations from all sectors of the water-data community including Federal, State, local governmental, academic, interstate, and private organizations. Foreign organizations are also invited to become affiliated with the program. This Directory provides the names, addresses, and telephone numbers of all NAWDEX member organizations and their designated NAWDEX representatives.

\section{NAWDEX MEMBERSHIP}

Membership in NAWDEX is voluntary and open to any water-oriented organization that wishes to take an active role. No dues or fees are associated with becoming a member. Members are required, however, to sign a memorandum of understanding with the NAWDEX Program Office that defines a member's general commitment to take an active role in NAWDEX activities, to provide information on its data holdings for indexing purposes, and to provide data from its holdings upon request. A sample copy of the memorandum of understanding is shown in the appendix. The contents of the memorandum of understanding may be amended, as necessary, to meet individual requirements of member organizations. 


\section{PURPOSE OF THE DIRECTORY}

This Directory identifies member organizations of NAWDEX and provides points of contact for NAWDEX activities within these organizations. In this manner, direct channels of communication are established between members, thereby improving communication throughout the water-data community. The Directory will be updated periodically to include new member organizations.

\section{ADDITIONAL INFORMATION}

Additional information concerning the NAWDEX program, membership, and services available may be obtained from:

\section{National Water Data Exchange}

U.S. Geological Survey

421 National Center

Reston, VA 22092

Telephone: (703) 648-6848

FTS $959-6848$ 
Acheron Engineering Services

40 Main Street

Newport, ME 04953

Designated Representative:

Gregory Brown

Telephone: (207) 368-5120

\section{Agricultural Research Service}

U.S. Dept. of Agriculture

Hydrology Laboratory, Room 101, Bldg 264

Beltsville, MD 20705

Designated Representatives:

Ralph Roberts

Computer Specialist

Telephone: (301) 344-4412

John W. Green
ANRE Department/USDA
Colorado State University
Fort Collins, CO 8052

Alabama Geological Survey

P.0. Box 0

Tuscaloosa, AL 35486

Designated Representative:

James D. Moore, Director, Water Resources Division

Telephone: (205) 349-2852

\section{Alameda County, Office of Education}

313 West Winton Avenue

Hayward, CA 94544-1198

Designated Representative:

Carolie s?y, Coordinator Science Project

Telephors: (415) 887-0152 ext. 345

\section{Alaska Department of Environmental Conservation}

State of Alaska

Department of Environmental Conservation

Pouch 0, Mail Stop 1800

Juneau, AK 99811

Designated " presentative:

Michae. A. Angelo, Systems Analyst

Telephone: 8-(907) 465-2621 
565 University

Fairbanks, AK 99701

Designated Representative:

Dr. Phylis K. Weber

Telephone: (907) 274-0516

\section{Alaska Department of Natural Resources}

Division of Geological and Geophysical Surveys

P.O. Box 772116

Eagle River, AK 99577

Designated Representative:

William E. Long

Chief, Water Resources Section

Telephone: (907) 696-0070

Alaska Department of Transportation and Public Facilities

Regional Hydrologist

Pouch 196900

Anchorage AK 99519-6900

Designated Representative:

W. F. (Skip) Barber

Telephone: (907) 333-0616 or $338-4200$

\section{Albert Mann Library - Cornell University}

Computer Files

Mann Library

Ithaca, NY 14853-4301

Designated Representative:

Katherine Chiang

Telephone: (607) 255-2199

Aqua Resources, Inc.

2030 Addison Street

Suite 500

Berkeley, CA 94704

Designated Representative:

David N. Church

Telephone: (415) 540-6954 
Aqua Terra Consultants

2672 Bayshore Parkway

Suite 1001

Mountain View, CA 94043

Designated Representative:

Brian R. Bicknell

Telephone: (415) 962-1864

Aquatic Systems Branch

U.S. Fish and Wildlife Service

National Ecology Research Center

4512 McMurray Avenue

Fort Collins, CO 80525-3400

Designated Representative:

Robert T. Milhous

Telephone: (303) 226-9331

FTS 323-5331

Arco Oil and Gas Company

2300 W. Plano Parkway

Plano, TX 75075

Designated Representative:

James R. Chatham

Telephone: (214) 754-6282

Arkansas Department of Pollution Control and Ecology

8001 National Drive

Little Rock, AR 72209

Designated Representative:

Jerry M. Baker, Data Base Analyst

Telephone: (501) 562-7444

Arkansas Soil and Water Conservation Commission

One Capitol Mall

Suite 2D

Little Rock, AR 72201

Designated Representative:

Jon Sweeney

Telephone: (501) 682-1611 
U.S. Army Corps of Engineers

DAEN - CWH - W

Washington, D.C. 20314

Designated Representatives:

Ming Tseng

Office of the Chief of Engineers

Telephone: (202) 272-8509

Steven Daly

72 Lyme Road

Hanover, NH 03755

Telephone: FTS 836-4100

James M. Robinson, Jr.

Norfolk District

803 Front Street

Norfolk, VA 23510-1096

Telephone: (804) 441-3774

FTS $827-3774$

Ayres Associates, Inc.

1300 West Clairemont Avenue

P.O. Box 1590

Eau Claire, WI 54702-1520

Designated Representative;

Jan Smit

Telephone: (715) 834-3161

BCA Environmental

1 Overlook Drive

Unit 16

Amherst, NH 03031

Designated Representative:

Stephen R. Clough

Telephone: (603) 672-2511

BCI Geonetics, Inc.

P.O. Box 529

Airport Road

Laconia, $\mathrm{NH} 03247$

Designated Representative:

Rose Marie de Mars

Telephone: (603) 528-1007
C. Richard Gowin

U.S. Army Engineer District Attn: ADP Center

P.O. Box 59

Louisville, KY 40201-0059

Telephone: (502) 582-5723

FTS 352-5723

Robert F. Gore

Baltimore District

P.O. Box 1715 (NABPL-S)

Baltimore, MD 21203

Telephone: (301) 962-4710 
BCM Eastern, Inc.

One Plymouth Meeting Mall

Plymouth Meeting, PA 19462

Designated Representative:

Robert M. Hardy, P.E.

Telephone: (215) 825-3800

Bechtel Corporation

50 Beale Street

P.0. Box 3965

San Francisco, CA 94119

Designated Representative:

Samuel L. Hui

Telephone: (415) 768-7734

Besha Associates Engineering Corporation

1 Washington Square

Albany, NY 12205

Designated Representative:

Christopher I. McGrew

Telephone: (518) 456-7712

Bhate Engineering Corporation

52175 th Avenue

South Birmingham, AL 35212-3515

Designated Representative:

John B. Milledge

Telephone: (205) 591-7062

Bingham Engineering

100 Lin̉dbergh Plaza 2

5160 Wiley Post Way

Salt Lake City, UT 84116

Designated Representative:

Alan B. Taylor

Telephone: (801) 532-2520 
Black and Veatch Engineers-Architects

P. O. Box 8405

Kansas City, MO 64114

Designated Representative:

Richard P. Byrne

Telephone: (913) 339-3624

Bonneville Power Administration (U.S.)

U.S. Department of Energy

P.O. Box 3621

Portland, OR 97208

Designated Representatives:

Michael Deatherage-Newsom (Technical matters)

Telephone: (503) 230-3643

FTS : $8-429-3957$

Bookman-Edmonston Engineering, Inc.

100 North Brand Blvd

Suite 600

Glendale, CA 91203

Designated Representative:

William O'Brien

Telephone: (818) 244-0117

Boyle Engineering Corporation

100 Howe Avenue

Suite 250 North

Sacramento, CA 95825

Designated Representatives:

David Mattern

Telephone: (916) 483-7771

Bromwe11 and Carrier, Inc.

202 Lake Miriam Drive

P.0. Box 5467

Lakeland, FL 33807

Designated Representative:

Wayne A. Ericson

Telephone: (813) 646-8591
Denver West--Building 19

165 South Union Boulevard

Suite 200

Lakewood CO 80228

Ray Bennett

Telephone: (303) 987-3443 
Brookhaven National Laboratory

Building 703M

Upton, NY 11973

Designated Representative:

Dr. Edward Kaplan

Telephone: (516) 282-2007

FTS 666-2007

Bureau of Indian Affairs (U.S.)

U.S. Department of the Interior Branch of Regional Water Rights Protection P.O. Box 26567

Albuquerque, NM 87125-6567

Designated Representative:

Jane G. Marshall

Telephone: (505) 766-3167

FTS 474-3167

\section{Bureau of Land Management (U.S.)}

U.S. Department of the Interior 18 th and C Streets, N.W.

Washington, D.C. 20240

Designated Representatives:

Daniel P. Muller

Branch of Soil, Water, and Air

Telephone: (202) 653-9210

FTS 653-9210

Colorado State office

Dave Harned

Engineer Branch (CO-941A)

2850 Youngfield Street

Lakewood, CO 80215-7076

Telephone: (303) 236.1741

FTS $776-1741$
Loi 0. Moe

Oregon State Office (94-C)

P.O. Box 2965

Portland, OR 97208

FTS: $8-429-6814$

Jack Dugwyler

Denver Federal Center

P.O. Box 25047

Denver, CO 80225-0047

Telephone: (303) 236-0202

FTS 776-0202

Division of Solid Minerals Operation, BLM

Dave E. Hoglund

Main Interior Bldg., Rm. 3411

Washington, DC 20240

Telephone: (202) 343-7753 
Names and addresses of the contacts for Bureau of Land Management State Offices (State Hydrologists) follow:

Lyle Linne11, Hydrologist

Division of Resources

BLM Alaska State Office

701 C Street, Box 13

Anchorage, AK 99513

Telephone: (907) 271-5069

FTS 399-0150 (ask operator

for) (907) $271-5069$

Dan Mcglothlin, Hydrologist

Division of Resources

BLM Arizona State office

3707 N. 7th Street

Phoenix, AZ 85014

Telephone: (602) 241-5509

FTS 261-5509

Karl Gebhardt, Hydrologist

Division of Resources

BLM Idaho State office

3380 Americana Terrace

Boise, ID 83720

Telephone: (208) 334-1892

FTS $554-1892$

Peter Bierbach, Hydrologist

Division of Lands and Renewable Resources

BLM Montana State Office

P.O. Box 36800

Billings, MT 59107

Telephone: (406) 657-6655

FTS 585-6655

Chuck Pettee, Hydrologist

Division of Resources

BLM New Mexico State office

P.O. Box 1449

Santa Fe, NM 87501

Telephone: (505) 988-6231

FTS 476-6231

Dick Jewel1, Hydrologist

Division of Resources

BLM Nevada State Office

P.O. Box 12000

Reno, NV 89520

Telephone: (720) 784-5455

FTS $470-5455$
State Office Hydrologist

Division of Resources

BLM California State office

Federal Office Building

2800 Cottage Way, Room E-2841

Sacramento, CA 95825

Telephone: (916) 484-4701

FTS 468-4701

Tom Hewitt, Hydrologist

Branch of Renewable Resources

BLM Colorado State office

Colorado State Bank Building

1600 Broadway, Room 700

Denver, CO 80202

Telephone: (303) 274-0070

FTS 274-0070

Loi 0. Moe

Division of Resources

BLM Oregon State Office

P.O. Box 2965

Portland, OR 97208

Telephone: (503) 231-2253

FTS 429-2253

Boyd Christensen, Hydrologist

Division of Resources

BLM Utah State office

324 South State Street

Salt Lake City, UT 84111-2303

Telephone: (801) 524-3120

FTS 588-3120

State Office Hydrologist

Division of Resources

BLM Eastern States Office

350 South Pickett Street

Alexandria, VA 22304

Telephone: (703) 235-2866

FTS 235-2866

Cliff Franklin, Hydrologist

Division of Resources

BLM Wyoming State Office

P.0. Box 1828

Cheyenne, WY 82001

Telephone: (307) 778-2220

FTS $328-2425$ 
Bureau of Recle: ation (U.S.)

U.S. Department of the Interior Denver Office

P.0. Box 25007

Code D-5755, Building 67

Denver, CO 80225

Designated Representatives:

Wayne Cheney

Telephone: (303) 236-3813

FTS 776-3813

Great Plains Region

P.O. Box 36900

Billings, MT 59107

Designated Representatives:

Gordon Aycock

Telephone: (406) 657-6416

FTS 585-6416

Burns and McDonnell

4800 East 63rd Street

P.O. Box 419173

Kansas City, MO 64141-0173

Designated Representatives:

Gene L. Foster

Telephone: (816) 333-4375

(Alternate: Dave Stous)

\section{CH2M Hill}

625 Herndon Parkway

P. 0. Box 4400

Reston, VA 22090

Designated Representatives:

John Glass, Water Resources

Telephone: (703) 471-1441

Nea1 Dixon

P.O. Box 2088

Redding, CA 96099

Telephone: (916) 243-5831
Bill Vernier

Planning Division

P.O. Box 11568

Salt Lake City, UT 84147
David Peterson

3840 Rosin Court

Suite 110

Sacramento, CA 95834

Telephone: (916) 920-0300 
Calexco, Inc.

2200 West Berry Avenue

Littleton, CO 80120

Designated Representative:

Clement A. Lehnertz, Jr.

Telephone: (303) 794-8389

\section{Calgon Corporation}

Route 60 at Campbell's Run Road

Pittsburgh, PA 15205

Designated Representive:

Betty P. Schwarz, Information Center

Telephone: (412) 777-8205

California Department of Water Resources

Division of Local Assistance

P.O. Box 942836

Sacramento, CA 94236-0001

Designated Representative:

Joseph Kick, Chief, Data Management

Telephone: (916) 327-1677

\section{Calpine Corporation}

2001 Gateway Place

San Jose, CA 95110

Designated Representative:

Howard A. Eriksen

Telephone: (408) 280-5811

Carolina Power \& Light Company

P.O. Box 1551

Raleigh, NC 27602

Designated Representative:

C. C. Wheeler, Senior Engineer

Telephone: (919) 546-6725 


\section{Carr/Associates}

P.0. Box 1158

Gig Harbor, WA 98335

Designated Representative:

Donald F. Kellum

Telephone: (206) 851-5562

C-b Site, Occidental Oil Shale, Inc.

20011 County Road 5

Rifle, CO 81650

Designated Representatives:

Susan Robinson

Telephone: (303) 878-4074

(Alternate: Fred W. Noble)

Center for Water Resources Research, Utah State University

Utah State University

Logan, UT 84322-8200

Designated Representative:

Dr. L. Douglas James, Director

Telephone: (801) 750-3168

Chas T. Main, Inc.

101 Huntington Avenue

Boston, MA 02199

Designated Representatives:

Dr. Chiranjib K. Sarkar

Telephone: (617) 262-3200

Chen-Northern, Inc.

1610 B Street

P.O. Box 4699

Helena, MT 59604

Designated Representative:

Patricia Bugosh

Telephone: (406) 449-3729 


\section{Christensen Development Corporation}

538 Silverado Drive

Tiburon, CA 94920

Designated Representative:

John P. Christensen

Telephone: (415) 435-2606

City of Aurora, Utilities Administration

1470 S. Havana Street

Aurora, CO 80012

Designated Representative:

Pat McDermott

Telephone: (303) 695-7278

City of Everett Engineering Department

3200 Cedar Street

Everett, WA 98201

Designated Representative:

Daniel J. Mathias, Asst. Engineer

Telephone: (206) 259-8811

City of Savannah Industrial and Domestic Water Supply

P.0. Box 4101

Port Wentworth, GA 31407

Designated Representative:

Harry Jue, Jr., Water Operations Director

Telephone: (912) 964-5115

Clement Associates, Inc.

9300 Lee Highway

Fairfax, VA 22031-1207

Designated Representative:

Andrew Warner

Telephone: (703) 934-3838 
Office of the State Engineer

1313 Sherman Street

Room 818

Denver, CO 80203

Designated Representatives:

Dr. Jeris A. Danielson, State Engineer

Will W. Burt

Telephone: (303) 866-3581

Colorado River Water Conservation District

P.O. Box 1120

Glenwood Springs, CO 81602

Designated Representative:

David H. Merritt

Telephone: (303) 945-8522

Colorado State University, Department of Agricultural and Chemical Engineering

Fort Collins, CO 80523

Designated Representative:

Dr. Jim Loftis

Telephone: (303) 491-7923

Computer Innovations

4213 Marvin Place

Raleigh, NC 27609

Designated Representative:

Melvin D. Edwards

Telephone: (919) 787-2627

Consolidated Hydro, Inc.

Two Greenwich Plaza

Greenwich, CT 06830

Designated Representative:

Ram H. SE inani, P.E.

Telephone: (203) 661-4203 
Council on Environmental Quality (U.S.)

722 Jackson Place, N.W.

Washington, DC 20503

Designated Representative:

John C. Jens

Telephone: (202) 395-5750

Department of Civil Engineering

University of California-Davis

206 Walker Hall

Davis, CA 95616

Designated Representatives:

Gerald T. Orlob

Telephone: (916) 752-1424

Department of Energy (U.S.)

Office of Environmental Analysis EH-22, 4G-036 Forrestal Building

Washington, DC 20585

Designated Representatives:

Sandy Guill

Office of Environmental Analysis

Steve M. Baker

Rockwell Hartford Operations

EH-22, 4G-036 Forrestal BIdg.

Telephone: (202) 586-4628

P.O. Box $800 /$ PBB333

FTS $896-4628$

Richland, WA 99352

Telephone: (509) 376-7981

FTS 444-7981

Department of Geology, San Diego State University

San Diego, CA 92182

Designated Representative:

Dr. David Huntley, Professor of Geological Sciences

Telephone: (619) 594-5483

Deuel and Associates, Inc.

7208 Jefferson Drive, NE

Albuquerque, NM 87109

Designated Representative:

Robert $W$. Prindle

Telephone: (505) 345-8732 


\section{Dewberry and Davis}

8401 Arlington Boulevard

Third Floor

Fairfax, VA 22031

Designated Representatives:

Chuck Wood

Telephone: (703) 849-0324

Dow Chemical, U.S.A. - Earth Sciences

400 W. Belt South

Houston, TX 77042

Designated Representative:

Laura M. Gammil1

Telephone: (713) 978-3818

Duke University, School of Engineering

Department of Civil Engineering

Durham, NC 27706

Designated Representative:

$\mathrm{Dr}$. Migue1 A. Medina, Jr.

Telephone: (919) 684-2434

\section{Dunn Geoscience Corporation}

12 Metro Park Road

Albany, NY 12205

Designated Representatives:

Paul Wm. Hare, Hydrogeologist

Telephone: (518) 458-1313

(Alternate: Thomas M. Johnson)

Dynamac Corporation

11140 Rockville Pike

Rockville, MD 20852

Designated Representative:

Satish C. Bhalla

Telephone: (301) 468-2500 
1325 N.W. 9th Street

Corvallis, OR 97330

Designated Representative:

Joseph A. Bernert

Telephone: (503) 758-1330

\section{Ebasco Environmental}

555 Capitol Mall

Suite 625

Sacramento, CA 95814

Designated Representative:

Richard M. Sitts

Telephone: (916) 442-5356

Ebasco Services, Inc.

10900 N. E. 8th Street, 4th floor

Bellevue, WA 98004

Designated Representatives:

Mark Killgore

Telephone: (206) 451-4596

Feh Fan Yeh

3000 West McArthur Boulevard

Santa Ana, CA 92704

Telephone: (714) 662-4000
Lissette Miguel

2 World Trade Center

New York, NY 10048

Telephone: (212) 839-2956

Edward E. Clark Engineers-Scientists, Inc.

11960 S. W. 144th Street

Miami, FL 33186

Designated Representative:

Edward E. Clark, President

Telephone: (305) 233-1411

Electric Power Research Institute

3412 Hillview Avenue

P.O. Box 10412

Palo Alto, CA 94303

Designated Representative:

Edward G. Altouney

Planning and Evaluation Division

Telephone: (415) 855-2626

FTS 8-(415)-855-2626 
Engineering Methods and Applications, Inc.

2999 Hartley Road

Suite 202

Jacksonville, FL 32257

Designated Representative:

Cheryl A. Johnson

Telehone: (904) 268-3205

Environmental Biology Research Program

Box 5187

Department of Biology

Tennessee Technological University

Cookeville, TN 38505

Designated Representatives:

Dr. Eric L. Morgan

Dr. R. Don Estes

Telephone: (615) $372-3401$ or 3134

Environmental Defense Fund

5655 College Avenue

Suite 304

Oakland, CA 94618

Designated Representative:

Deborah Moore

Telephone: (415) 658-8008

Environmental Protection Agency (U.S.)

401 M Street

Washington, DC 20460

Designated Representatives:

Philip L. Taylor, Chief

Environmental Analysis Section

Telephone: (202) 382-7046

Fred Leutner

Telephone: (202) 382-7040

Joseph A. Sierra, Acting Chief

Client Services Branch

TM228

1505 Planning Research Drive

Mclean, VA 22102

Telephone: (703) 883-8861

Kenneth Scarbrough

EMSL, P.O. Box 93478

Las Vegas, NV 89193-3478

Telephone: (702) 798-2645 
Environmental Protection Agency, Region III

Groundwater Protection Section

841 Chestnut Street

Philadelphia, PA 19107

Designated Representative:

Stuart Kerzner, Chief, GWP Section

Telephone: (215) 597-8826

Environmental Protection Agency, Region IV

345 Courtland Street

Atlanta, GA 30365

Designated Representative:

Leonard Nowak

Telephone: (404) 347-2126

FTS 257-2126

Environmental Protection Agency, Region V

230 South Dearborn Street

Chicago, IL 60604

Designated Representative:

Stuart C. Ross

Data Processing Branch 5MSD-11

Telephone: (312) 353-0299

FTS 353-0299

Environmental Protection Agency, Region VII

726 Minnesota Avenue

Kansas City, KS 66101

Designated Representative:

J. Patrick Costello

Ground Water Protection

Telephone: (913) 236-2815

Environmental Protection Agency, Region X

1200 Sixth Avenue

MD - 103

Seattle, WA 98101

Designated Representative:

Jim Peterson

Telephone: (206) 442-2977

FTS 399-2977 
Environmental Research and Technology, Inc.

P.0. Box 2105

1716 Heath Parkway

Fort Collins, CO 80522

Designated Representative:

Peter Shanahan, Water Resources Specialist

Telephone: (303) 493-8878, ext. 228

Environmental Resources Management, Inc.

855 Springdale Drive

Exton, PA 19341

Designated Representative:

Librarian

Telephone: (215) 534-3500

Espey, Huston \& Associates, Inc.

916 Capital of Texas Highway South

P.O. Box 519

Austin, TX 78767

Designated Representative:

Ron Boyd

Telephone: (512) 327-6840

FMC Corporation

1801 California Street

Suite 2720

Denver, CO 80202

Designated Representative:

Chi I. Huang

Telephone: (303) 295-7391

Federal Energy Regulatory Commission

Office of Hydropower Licensing

825 North Capitol Street

Washington, DC 20426

Designated Representative:

Ed Melisky

Telephone: (202) 357-0657 
Federal Highway Administration

U.S. Department of Transportation 610 East 5th Street

Vancouver, WA 98661-3893

Designated Representatives:

Mark C. Browning

Telephone: FTS 422-7767

Fish and Wildlife Service (U.S.)

Division of Engineering

Lloyd 500 Building

Suite 1692

500 N.E. Multnomah Street

Portland, OR 97232

Designated Representatives:

Richard A. Johnson

Telephone: (503) 231-6145

FTS 429-6145

Fred T. Werner

Clear Lake Field office

17629 El Camino Real

Suite 211

Houston, TX 77058

Telephone: FTS 526-6700
Loudoun Tech Center

21400 Ridgetop Circle

Sterling, VA 22170

Brian Roberts

Telephone: (703) 285-0065

New England Field Office

400 Ralph Pill Market Place, Bridge Street

Concord, NH 03001

Joe F. Mckeon

Telephone: (603) 225-4411

FTS $834-4411$

See also: Aquatic Systems Branch; National Ecology Research Center; and National Fisheries Contaminant Research Center

Florida Department of Environmental Regulation

Twin Towers Office Building

2600 Blair Stone Road, Room A115

Tallahassee, FL 32399-2400

Designated Representative:

Spencer Lepley

Bureau of Information Systems

Telephone: (904) 488-0892

Florida Institute of Phosphate Research

1855 West Main Street

Bartow, FL 33830

Designated Representative:

Betty Faye Stidham, Librarian

Telephone: (813) 533-0983 


\section{Forest Service (U.S.)}

U.S. Department of Agriculture

P.O. Box 96090

Washington, DC 20090

Designated Representatives:

Rhey Solomon

Telephone: (703) 235-8178

Gordon Stuart

Dean Knighton

Forest Environmental Research

Telephone: (703) 235-1071

Cooperative Forestry

Telephone: (703) 235-1289

Directors of Soil, Watershed, and Air Management

Region 1 Ron Haag

Federal Building

P.O. Box 7669

Missoula, MT 59807

Region 2 Charles J. Hendricks 11177 West 8th Avenue Box 25127

Lakewood, CO 80225

Region 3 Noel Larson, Director Watershed and Air Mgmt. 517 Gold Avenue, S.W. Albuquerque, NM 87102

Region 4 Ray Hall

Federal Building 32425 th Street Ogden, UT 84401
Region 5 Andrew A. Leven

630 Sansome Street

San Francisco, CA 94111

Region 6 Ecology, Range, \& Watershed 319 S.W. Pine Street Box 3623

Portland, OR 97208

Region 8 Keith Grest

Soil, Water, and Air

1720 Peachtree Road, N.W. Atlanta, GA 30309

Region 9 Harry Parrott

USDA Forest Service 310 West Wisconsin Avenue Milwaukee, WI 53203

Region 10 Regional Hydrologist USDA Forest Service Box 1628

Juneau, AK 99802-1628 
Forest Service Research Stations

\section{Location}

North Central Station

Northeastern Station

Pacific Northwest Station

Rocky Mountain Station

Coweeta Hydrologic Laboratory

Southern Station

Location

Northeastern Area
Name

Station Director

Station Director

Station Director

Station Director

Director

Station Director

\section{Address}

USDA Forest Service 1992 Folwell Avenue St. Paul, MN 55108

USDA Forest Service 5 Radnor Corporate Center 100 Matsonford Road Suite 200

Radnor, PA 19087

USDA Forest Service P.O. Box 3890

Portland, OR 97208

USDA Forest Service 1960 Addison Street Box 245

Berkeley, CA 94701

USDA Forest Service 240 West Prospect St.

Fort Collins, CO 80526

Southeastern Forest Experiment Station

Route 1, Box 216 Otto, NC 28763

USDA Forest Service U.S. Postal Service Building 701 Loyola Avenue

New Orleans, LA 70113

State and Forestry Areas

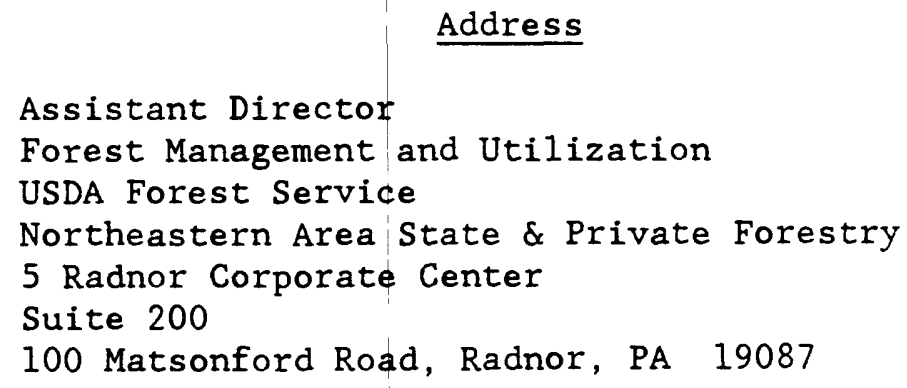

Address

Assistant Director

Forest Management and Utilization USDA Forest Service Northeastern Area State \& Private Forestry 5 Radnor Corporate Center Suite 200 100 Matsonford Road, Radnor, PA 19087 
State and Forestry Areas--Continued

Southeastern Area

$$
\begin{aligned}
& \text { Director, Soil, Water, and Air Unit } \\
& \text { USDA Forest Service } \\
& 1720 \text { Peachtree Road, NW, Room } 846 \mathrm{~N} \\
& \text { Atlanta, GA } 30367
\end{aligned}
$$

Fort Dodge Department of Public Works

Municipal Building

Fort Dodge, IA 50501

Designated Representative:

Frank F. Farmer, Public Works Director

Telephone: (515) 576-3601

\section{GAI Consultants}

570 Beatty Road

Monroeville, PA 15146

Designated Representative:

Craig D. Martin

Telephone: (412) 856-6400

GANCOM, Inc.

P.O. Box 1459

Harrisburg, PA 17105-1459

Designated Representative:

David L. Sprow, Vice President

Telephone: (717) 763-7226

Geauga County Office of Sanitary Engineer, (Ohio)

237 Main Street

Chardon, OH 44024

Designated Representative:

Zane F. Lee, County Sanitary Engineer

Telephone: (419) 285-2222, ext. 141-143

General Sciences Corporation

6100 Chevy Chase Drive

Suite 200

Laure 1, MD 20707

Designated Representative:

Stuart H. Wollman

Telephone: (301) 953-2700 
U.S. Department of the Interior

Water Resources Division

National Center

12201 Sunrise Valley Drive

Reston, VA 22092

Designated Representatives:

William B. Mann, IV

Asst. Chief Hydrologist for Operations

441 National Center

Telephone: (703) 648-5031

FTS $959-5031$

Designated Representatives of the USGS Water Resources Division, (WRD)

District Offices:

D. Briane Adams

District Chief, WRD

U.S. Geological Survey

52019 th Avenue

Tuscaloosa, AL 35401

Telephone: (205) 752-8104

FTS 229-1061

Philip A. Emery

District Chief, WRD

U.S. Geological Survey

4230 University $\mathrm{Dr}$, Suite 201

Anchorage, AK 99508-4664

Telephone: (907) 271-4138

FTS $8-(907)-271-4138$

Robert D. MacNish

District Chief, WRD

U.S. Geological Survey

300 West Congress Street, FB-44

Tucson, AZ 85701

Telephone: (602) 629-6671

FTS $762-6671$
Charles A. Pascale

District Chief, WRD

U.S. Geological Survey

Building 53, Denver Federal Center

Lakewood, CO 80225

Telephone: (303) 236-4882

FTS $776-4882$

Chester E. Thomas, Jr.

Chief, Connecticut office, WRD

U.S. Geological Survey

Room 525, Abraham A. Ribicoff

Federal Building

450 Main Street

Hartford, CT 06103

Telephone: (203) 240-3060

FTS 244-3060

Irwin H. Kantrowitz

District Chief, WRD

U.S. Geological Survey

Hobbs Federal Bldg.

Suite 3015

227 N. Bronough

Tallahassee, FL 32301

Telephone: (904) 681-7631

FTS $965-7631$ 
Ector E. Gann

District Chief, WRD

U.S. Geological Survey

Room 2301, Federal office Building

700 West Capitol Avenue

Little Rock, AR 72201

Telephone: (501) 378-6391

FTS $740-6391$

John M. Klein

District Chief, WRD

U.S. Geological Survey

Federal Bldg., Rm. W-2234

2800 Cottage Way

Sacramento, CA 95825

Telephone: (916) 978-4633

FTS $460-4633$

Jerry L. Hughes

District Chief, WRD

U.S. Geological Survey

230 Collins Road

Boise, ID 83702

Telephone: (208) $334-1750$

FTS $554-1750$

Richard Novitsky

District Chief, WRD

U.S. Geological Survey

Busey Bank County Plaza

102 E. Main Street, 4th Floor

Urbana, IL 61801

Telephone: (217) 398-5354

FTS $958-5354$

Dennis K. Ste art

District Chief, WRD

U.S. Geological Survey

5957 Lakeside Boulevard

Indianapolis, IN 46278

Telephone: (317) 290-3333

FTS 335-3333
Jeffrey $T$. Armbruster

District Chief, WRD

U.S. Geological Si vey

6481 Peachtree Industrial Blvd.

Suite B

Doraville, GA 30360

Telephone: (404) 986-6860

FTS 257-6860

William Meyer

District Chief, WRD

U.S. Geological Survey

677 Ala Moana Boulevard

Suite 415

Honolulu, HI 96813

Telephone: (808) 546-2653

FTS $8-(808)-546-2653$

Alfred L. Knight

District Chief, WRD

U.S. Geological Survey

2301 Bradley Avenue

Louisville, KY 40217

Telephone: (502) 582-5241

FTS $352-5241$

Darwin D. Knochenmus

District Chief, WRD

U.S. Geological Survey

P.O. Box 66492

6554 Florida Boulevard

Baton Rouge, LA 70806

Telephone: (504) 389-0281

FTS 687-0281

Herbert J. Freiberger

District Chief, WRD

U.S. Geological Survey

208 Carroll Building

8600 La Salle Road

Towson, MD 21204

Telephone: (301) 828-1535

FTS $922-7872$ 
USGS Water Resources Division District Offices--Continued

Richard A. Engberg

District Chief, WRD

U.S. Geological Survey

P.O. Box 1230

Room 269 Federal Building

400 South Clinton Street

Iowa City, IA 52244

Telephone: (319) 337-4191

Thomas $L$. Huntzinger

District Chief, WRD

U.S. Geological Survey

4281 Quail Crest Place

Lawrence, KS 66044

Telephone: (913) 864-4321

William J. Herb

District Chief, WRD

U.S. Geological Survey

Room 702, Post office Building

St. Paul, MN 55101

Telephone: (612) 725-7841

FTS $725-7841$

Michael W. Gaydos

District Chief, WRD

U.S. Geological Survey

Suite 710

Federal Office Building

100 West Capitol Street

Jackson, MS 39269

Telephone: (601) 965-4600

FTS 490-4600

Daniel P. Bauer

District Chief, WRD

U.S. Geological Survey

1400 Independence Road

Mail Stop 200

Rolla, MO 65401

Telephone: (314) 341-0825
Ivan C. James, II

District Chief, WRD

U.S. Geological Survey

150 Causeway Street, Suite 1309

Boston, MA 02114-1384

Telephone: (617) 565-6860

FTS $835-6860$

T. Ray Cummings

District Chief, WRD

U.S. Geological Survey

6520 Mercantile Way, Suite 5

Lansing, MI 48911

Telephone: (517) 377-1608

FTS 374-1608

William J. Carlswell

State office Chief, WRD

U.S. Geological Survey

Room 224, Federal Building

705 North Plaza Street

Carson City, NV 89701

Telephone: (702) 882-1388

Donald E. Vaupel

District Chief, WRD

U.S. Geological Survey

Suite 206

Mountain View office

810 Bear Tavern Road

Trenton, NJ 08628

Telephone: (609) 771-3900

Robert L. Knutilla

District Chief, WRD

U.S. Geological Survey

Pinetree Office Park, Suite 200

4501 Indian School Road, N.E.

Albuquerque, NM 87110

Telephone: (505) 262-6630

FTS $474-6630$ 
USGS Water Resources Division District Offices--Continued

Joe A. Moreland

District Chief, WRD

U.S. Geological Survey

Federal Building

Drawer 10076

Room 428, 301 South Park Avenue

Helena, MT 59626

Telephone: (406) 449-5263

FTS 585-5263

Michael Shulters

District Chief, WRD

U.S. Geological Survey

Room 406, Federal Building \&

U.S. Courthouse

100 Centennial Mall, North

Lincoln, NE 68508

Telephone: (402) 437-5155

FTS 541-5155

William F. Horak

District Chief, WRD

U.S. Geological Survey

821 East Interstate Avenue

Bismarck, ND 58501-1199

Telephone: (701) 255-4011, ext. 601 FTS 783-4610

Steven M. Hindall

District Chief, WRD

U.S. Geological Survey

975 West Third Avenue

Columbus, $\mathrm{OH} 43212$

Telephone: (614) 469-5553

FTS 943-5553

Harvel L. Case

District Chief, WRD

U.S. Geological Survey

Room 621

215 Dean A. McGee Avenue

Oklahoma City, OK 73102

Telephone: (405) 231-4256

FTS $736-4256$
L. Grady Moore

District Chief, WRD

U.S. Geological Survey

Room 343, U.S. Post Office \&

Courthouse

P.O. Box 1669

Albany, NY 12201

Telephone: (518) 472-3107

FTS $562-3107$

James F. Turner

District Chief, WRD

U.S. Geological Survey

Room 436, Century Postal Station P.O. Box 2857

Raleigh, NC 27602

Telephone: (919) 856-4510

FTS $672-4510$

Allen L. Zack

District Chief, WRD

U.S. Geological Survey

G.P.O. Box 4424

San Juan, PR 00936

Telephone: (809) 753-4660

FTS $809-749-4346$

Rodney N. Cherry

District Chief, WRD

U.S. Geological Survey

Strom Thurmond Federal Building

1835 Assembly Street, Suite 677A

Columbia, SC 29201

Telephone: (803) 765-5966

FTS $677-5966$

Richard E. Fidler

District Chief, WRD

U.S. Geological Survey

Room 317, Federal Building

200 4th Street, S.W.

Huron, SD 57350

Telephone: (605) 353-7176 
USGS Water Resources Division District Offices--Continued

Marvin 0. Fretwe11

Chief, Portland Office

Oregon State office

U.S. Geological Survey

10615 S.E. Cherry Blossom Drive

Portland, OR 97216

Telephone: (503) 231-2008

FTS 429-2011

David E. Click

District Chief, WRD

U.S. Geological Survey

P.0. Box 1107

4 th Floor, Federal Building

228 Walnut Street

Harrisburg, PA 17108

Telephone: (717) 782-4514

FTS 590-4514

\section{Vacant}

District Chief, WRD

U.S. Geological Survey

1016 Administration Bldg.

1745 West 1700 South

Salt Lake City, UT 84104

Telephone: (801) 524-5663

FTS 588-5663

Gary S. Anderson

District Chief, WRD

U.S. Geological Survey

3600 West Broad Street, Room 606

Richmond, VA 23230

Telephone: (804) 771-2427

FTS $925-2427$

Gerald G. Parker, Jr.

District Chief, WRD

U.S. Geological Survey

1201 Pacific Avenue

Suite 600

Tacoma, WA 98402

Telephone: (206) 593-6510

FTS 390-6510
Ferdinand Quinones-Marquez

District Chief, WRD

U.S. Geological Survey

A-413 Federal Building

U.S. Courthouse

Nashville, TN 37203

Telephone: (615) 736-5424

FTS $852-5424$

Charles R. Burchett

District Chief, WRD

U.S. Geological Survey

8011 Cameron Road

Building 1

Austin, TX 78701

Telephone: (512) 832-5791

David H. Appel

District Chief, WRD

U.S. Geological Survey

603 Morris Street

Charleston, WV 25301

Telephone: (304) 347-5130

FTS $930-5132$

Vernon W. Norman

District Chief, WRD

U.S. Geological Survey

6417 Normandy Lane

Madison, WI 53719

Telephone: (608) 274-3801

FTS 276-3801

James E. Kircher

District Chief, WRD

U.S. Geological Survey

P.O. Box 1125

J.C. O'Mahoney Federa1 Center

2120 Capitol Avenue, Room 4006

Cheyenne, WY 82003

Telephone: (307) 772-2153

FTS $328-2153$ 
GeoResearch, Inc.

2815 Montana Avenue

Billings, MT 59101

Designated Representatives:

Douglas Richardson

Dan Erikson

Telephone: (406) 248-6771

Geraghty \& Miller, Inc.

900 Massachusetts

Suite 600

Lawrence KS, 66044

Designated Representatives:

Office Manager

Telephone: (913) 841-7641

Gidley Laboratories, Inc.

Fairhaven, MA 02719

Designated Representative:

Dr. Philip T. Gidley, President

Fairhaven, MA 02719

Gill and Pulver Engineers, Inc.

1824 Tribute Road

Suite A

Sacramento, CA 95815

Designated Representative:

John W. Pulver, P.E.

Telephone: (916) 920-4717

Greenhorne \& 0'Mara, Inc.

9001 Edmonston Rd

Greenbelt, MD 20770

Designated Representative:

John M. Crouse

Telephone: (301) 982-2800
7800 Castleton Street

Suite 175

City of Industry, CA 91748

N. Thomas Sheahan

Telephone: (818) 965-4048 
Greiner, Inc.

570 West 44 th Avenue

Denver, CO 80216

Designated Representative:

Tyler Smart, Department Manager (Hydrology)

Telephone: (303) 455-7321

HRS Water Consultants, Inc.

Union Plaza Building

200 Union Boulevard

Suite 400

Lakewood, co 80228

Designated Representative:

Robert D. Tafelski, President

Telephone: (303) 989-2837

Handar, Inc.

2923 Olney-Sandy Spring Road

Suite E.

Olney, MD 20832

Designated Representative:

Gary J. Kemp

Telephone: (301) 774-1616

\section{Harmsworth Associates}

23232 Peralta Drive

Suite 209

Laguna Hills, CA 92653

Designated Representative:

Rodney Harmsworth

Telephone: (714) 855-0222

Hazen and Sawyer, P.C.

4000 Westchase Boulevard

Suite 550

Raleigh, NC 27607

Designated Representative:

Jim McCarthy

Telephone: (919) 833-7152 
Herbert E. Skibitzke, P.E.

P.0. Box 27334

Tempe, AZ 85285-7334

Designated Representative:

Herbert E. Skibitzke

Telephone: (602) 730-9432

Hetch Hetchy Water and Power

1155 Market Street

San Francisco, CA 94103

Designated Representatives:

A. B. Moran, General Manager

Telephone: (415) 554-0725

(Alternate: L. T. Klein, Deputy General Manager)

Hittman Ebasco Associates, Inc.

9151 Rumsey Road

Columbia, MD 21045

Designated Representative:

Steven A. Schaffer

Telephone: (301) 730-8525

Honolulu Board of Water Supply

City and County of Honolulu

630 South Beretania Street

Honolulu, HI 96843

Designated Representative:

Kazu Hayashida, Manager and Chief Engineer

Telephone: (808) 527-6180

Hydro Research Science

3334 Victor Court

Santa Clara, CA 95054

Designated Representative:

Nancy S. Fadis

Telephone: (408) 988-1027 
Hydro-Triad LTD

1310 Wadsworth Boulevard

Suite 100

Lakewood, CO 80215

Designated Representative:

Jay Nelson

Telephone: (303) 238-6022

Hydromet Data Services, Inc.

9685 Main Street

P.O. Box 710

Brewerton, NY 13029

Designated Representative:

Neal J. Gruber

Telephone: (315) 676-2657

Illinois Department of Transporation

Division of Water Resources

2300 S. Dirksen Parkway

Springfield, IL 62764

Designated Representative:

Melvin Allison, P.E.

Telephone: (217) 782-4636

Illinois State Water Survey

2204 Griffith Drive

Champaign, IL 61820

Designated Representative:

Robert A. Sinclair

Telephone: (217) 333-4952

In-Situ, Inc.

7401 W. Mansfield Avenue

Suite 114

Lakewood, CO 80235

Designated Representative:

Dr. Timothy D. Steele

Telephone: (303) 987-1877 
International Boundary and Water Commission

4171 Mesa, B1dg. \#C-310

E1 Paso, TX 79902

Designated Representative:

Andrew D. Seiger

Telephone: (915) 534-6704

International Ground Water Modeling Center (IGWMC)

Holcomb Research Institute

Butler University

4600 Sunset Avenue

Indianapolis, IN 46208

Designated Representative:

Stan Williams

Telephone: (317) 283-9458

Interstate Commission on the Potomac River Basin

6110 Executive Boulevard

Suite 300

Rockville, MD 20852-3903

Designated Representative:

Carlton Haywood

Telephone: (301) 984-1908

Iowa Department of Natural Resources, Geological Survey Bureau

123 North Capitol Street

Iowa City, IA 52242

Designated Representative:

Richard L. Talcott

Telephone: (319) 338-1173

Iowa Institute of Hydraulic Research

The University of Iowa

Iowa City, IA 52242

Designated Representative:

Konstantine Georgakakos, Associate Professor

Telephone: (319) 335-5222 
JBS Engineering

311 D Street

Suite A

West Sacramento, CA 95605

Designated Representative:

Stan Malinsky, Senior Engineer

Telephone: (916) 372-0534

J. Venn Leeds, Jr.

10807 Atwe 11

Houston, TX 77096

Designated Representative:

$\mathrm{J}$. Venn Leeds, Jr.

Telephone: (713) 723-4485

J. R. Nuckols, Ph.D., C.P.H.

5835 CR 52E

Bellvue, CO 80512

Designated Representative:

J. R. Nuckols

Telephone: (303) 491-7038

J.W. Patterson \& Associates, Inc.

6825 East Tennessee Avenue

Building 2, Suite 250

Denver, CO 80224

Designated Representative:

John W. Patterson, President

Telephone: (303) 320-1295

James M. Montgomery Consulting Engineers, Inc.

2328 10th Avenue, North

Suite 5A

Lakeworth, FL 33461

Designated Representatives:

Patrick J. Gleason

Telephone: (305) 586-8830
30 Corporate Park

Suite 310

Irvine, CA 92714

Robert Seeman

Telephone: (714) 261-7210 
John C. Landon \& Associates, Inc.

1454 U.S. 19 South

Suite 410

Clearwater, FL 34624

Designated Representative:

Steven J. Stuebs

Telephone: (813) 530-9709

Johnson \& Anderson, Inc.

Consulting Engineers

4479 Dixie Highway

Drayton Plains, MI 48020

Designated Representative:

John Emig, Jr.

Telephone: (313) 334-9901

Joint Montana University, Water Research Center

Room 412, Cobleigh Hall

Montana State University

Bozeman, MT 59717

Designated Representative:

Dr. Howard Peavy, Director

Telephone: (406) 994-6690

Jones and Stokes Associates

1725 23rd Street

Sacramento, CA 95816

Designated Representative:

Doug L. Brewer

Telephone: (916) 444-5638

K. M. $\mathrm{Ng}$ and Associates, Inc.

6243 Interstate 10 West

Suite 200

San Antonio, TX 78201

Designated Representative:

Razi Hosseini, Project Manager

Telephone: (512) 736-6623 
Kansas State Board of Agriculture

Division of Water Resources

109 Southwest Ninth Street

Suite 202

Topeka, KS 66612-1283

Designated Representative:

David L. Pope, P.E.

Chief Engineer/Director

Telephone: (913) 296-3717

Kearney Wastewater Treatment Plant

39 Central Avenue

South Kearney, NJ 07032

Designated Representative:

Larry Angelo, Chief Operator

Telephone: (201) 589-7599

Kentucky Department of Environmental Protection

Division of Water

18 Reilly Road - Frankfort Office Park

Frankfort, KY 40601

Designated Representative:

David Leist

Telephone: (502) 564-3410

Kindle, Stone \& Associates, Inc.

P.0. Box 1552

Longview, TX 75606

Designated Representative

Joe N. Harle

Telephone: (214) 297-7700

King County Department of Public Works

Surface Water Management Division

Dexter Horton Building - 7th Floor

Seattle, WA 98104

Designated Representative:

Jim Kramer

Telephone: (206) 296-6585 
107 North Lake Avenue

Tavares, FL 32778

Designated Representative:

Mr. William C. Davis, Executive Director

Telephone: (904) 343-3777

Lake Mary Water Department

P.O. Box 715

Lake Mary, FL 32746

Designated Representative:

Jim Orioles

Telephone: (305) 323-7221

Law Environmenta1, Inc.

112 Townpark Drive

Kennesaw GA 30144-5599

Designated Representatives:

Gerald E. Seaburn

Telephone: (404) 421-3400

(Alternate: Larry Neal)

\section{Lawrence Berkeley Laboratory}

University of California

Applied Science Division 90/3125

Berkeley, CA 94720

Designated Representative:

Ronald Ritschard

Telephone: (415) 486-6328

FTS $451-6328$

Lawrence Livermore National Laboratory

University of California

Livermore, CA 94550

Designated Representative:

Kenneth D. Pimentel

Telephone: (415) 422-0916
5510 Gray Street Suite 118

Tampa, FL 33609

Thomas F. Drexhase

Telephone: (813) 289-9491 
Leonard Rice Consulting Water Engineers, Inc.

2401 Fifteenth Street

Suite 300

Denver, CO 80202-1143

Designated Representative:

Leonard Rice

Telephone: (303) 455-9589

Los Angeles County Department of Public Works

P.O. Box 1460

Alhambra, CA 91802-1460

Designated Representative:

Ray Izadi

Telephone: (818) 458-6162

Louisville Water Company

435 South Third Street

Louisville, KY 40202

Designated Representative:

James C. Smith, Research Engineer

Telephone: (502) 569-3600, ext. 414

Maine Geological Survey

State of Maine Department of Conservation Station 22

Augusta, ME 04333

Designated Representative:

Troy T. Smith

Telephone: (207) 289-2801

Marin Municipal Water District

220 Nellen Avenue

Corte Madera, CA 94925

Designated Representative:

Glenn Young

Telephone: (415) 924-4600, ext. 238 
Marine Resources Council

Florida Institute of Technology

$150 \mathrm{~W}$. University Avenue

Melbourne, FL 32901

Designated Representative:

Diane D. Barile

Telephone: (407) 768-8000, ext. 8135

Maryland Natural Resources, Water Resources Administration

Water Rights Division

Tawes State Office Building, D-3

Annapolis, MD 21401

Designated Representaive:

Gary T. Setzer

Telephone: (301) 974-3675

McCall-Ellingson \& Morrill, Inc.

1721 High Street

Denver, CO 80218

Designated Representative:

Lyman R. Flook, Jr.

Telephone: (303) 321-2282

McLaren Environmental Engineering

11101 White Rock Road

Rancho Cordova, CA 95670

Designated Representative:

Frederick R. McLaren

Telephone: (916) 638-3696

Merced Irrigation District

P.O. Box 2288

Merced, CA 95344-0288

Designated Representative:

Thomas Reta, Chief Engineer/Manager

Telephone: (209) 722-5761 
Metropolitan Denver Sewage Disposal District No. 1

6450 York Street

Denver, CO 80229

Designated Representative:

Ronald D. French

Telephone: (303) 289-5941

Metropolitan District Water Bureau (Hartford, CT)

P.O. Box 800

555 Main Street

Hartford, CT 06101

Designated Representative:

Theodore Jaskulski

Telephone: (203) 278-7850

(Alternate: Claude A. Garritt)

\section{Millipore Corporation}

Ashby Road

Bedford, MA 01730

Designated Representative:

Susan Mansur

Manager, Information Center

Telephone: (617) 275-9200

Minnesota Department of Health, Division of Environmental Health

P.0. Box 9441

717 Delaware Street, S.E.

Minneapolis, MN 55440

Designated Representative:

Mr. Gary L. Englund, Chief

Section of Water Supply and General Engineering

Telephone: (612) 623-5330

Minnesota Pollution Control Agency

520 Lafayette Road

St Paul, MN 55155

Designated Representative

Kathy Svanda

Water Monitoring and Data Management

Program Development Section

Division of Water Quality

Telephone: (612) 296-8856 
P.0. Box 5348

2525 North West Street

Jackson, MS 39216

Designated Representative:

Carolyn Woodley

Telephone: (601) 354-6228

Mississippi Bureau of Land and Water Resources

P.O. box 10631

Jackson, MS 39209

Designated Representative:

James Hoffman

Telephone: (601) 961-5205

FTS 490-4399

Mississippi State University

P.O. Drawer CE

Mississippi State, MS 39762

Designated Representative:

Dr. Victor L. Zitta, Professor

Civil Engineering Department

Telephone: (601) 325-3050

(Alternate: Dr. Robert M. Scholtes)

Missouri Division of Geology and Land Survey

State Department of Natural Resources

P.0. Box 250

Rolla, MO 65401

Designated Representative:

Don E. Miller, Chief, WRD

Telephone: (314) 364-1752

Monroe County (NY) Water Authority

4799 Dewey Avenue

Rochester, NY 14612

Designated Representative:

James Nugent, Assistant Chemist

Telephone: (716) 621-1200 
Monsanto Agricultural Company

700 Chesterfield Village Parkway

St. Louis, MO 63198

Designated Representative:

David Gustafson

Telephone: (314) 537-6882

Montana Bureau of Mines and Geology

Montana Tech

Butte, MT 59701

Designated Representative:

Marvin R. Miller, Chief

Hydrology Division

Telephone: (406) 496-4155

Montana Department of Natural Resources and Conservation

Engineering Bureau

Water Resources Division

1520 E. 6th Avenue

Helena, MT 59620-2301

Designated Representative:

Melvin McBeath

Telephone: (406) 444-6646

Montgomery County Department of Environmental Protection

101 Monroe Street

Rockville, MD 20850

Designated Representative:

David W. Lake, Senior Environmental Engineer

Division of Environmental Planning and Monitoring

Telephone: (301) 217-2380

Morrison-Knudsen Engineers, Inc.

Headquarters Office

180 Howard Street

San Francisco, CA 94105

Designated Representatives:

Price E. Stiffler, Chief

Engineering Systems

Telephone: (415) 442-7411
Eastern District Office

70 Center Street, 2nd Floor Portland, ME 04101

Gregg E. Carrington

Telephone: (207) 774-1227 
108 East Wade

Mountain Home, AR 72653

Designated Representative:

Tommy J. Snow

Telephone: (501) 425-9565

Multitech, Inc.

P.0. Box 4078

Butte, MT 59702

Designated Representative:

David Tuesday, Hydrogeologist

Telephone: (406) 723-8213

(Alternate: Bill Bullock)

N.U.S. Corporation

1927 Lakeside Parkway

Suite 614

Tucker, GA 30084

Designated Representatives:

Lawrence Shelton

Telephone: (404) 938-7710
1300 N. 17th Street

Suite 1320

Arlington, VA 22209

Ross A. Dimmick

Telephone: (703) 522-8802

National Ecology Research Center - Leetown

U.S. Fish and Wildlife Service

Box 705

Kearneysville, WV 25430

Designated Representative:

Dr. James M. Brown

Telephone: (304) 725-2061

FTS 925-5282

National Fisheries Contaminant Research Center

U.S. Fish and Wildlife Service

Route 2, 4200 New Haven Road

Columbia, MO 65201

Designated Representatives:

El1-Piret Multer

Telephone: (314) 875-5399

FTS 276-5399

(Alternate: Chris Schmitt) 
6001 Executive Boulevard

Rockville, MD 20852

Telephone: (301) 443-8910

Designated Representatives of all NOAA Divisions:

Dr. Michael D. Hudlow, W/OH

Director, Office of Hydrology

National Weather Service, NOAA

806013 th Street

Silver Spring, MD 20910

Telephone: (301) 427-7658

Steacy D. Hicks

N/OMA1, Room 315

National Ocean Service, NOAA

6001 Executive Blvd.

Rockville, MD 20852

David F. McGinnis, Jr.

NESDIS/LSB (E/RA12)

NOAA/National Environmental Satellite, Data and Information Service

World Weather Building, Room 712

Washington, DC 20233

Telephone: (301) 763-8042

FTS 763-8042
Eugene A. Stallings

Chief, Hydrologic Services Branch, W/OH 22

National weather Service, NOAA 806013 th Street

Silver Spring, MD 20910

Telephone: (301) 427-7624

Addresses of Divisions of NOAA:

National Weather Service

8060 13th Street Room 504

Silver Spring, MD 20910

Telephone: (301) 427-7658

National Marine Fisheries Service

3300 Whitehaven Street, N.W.

Washington, DC 20235

Telephone: (202) 634-7283
National Ocean Service 6001 Executive Boulevard Rockville, MD 20852

Telephone: (301) 443-3778

Environmental Research Laboratories 325 South Broadway

Boulder, CO 80303

Telephone: (303) 497-5565

Great Lakes Environmental Research Laboratory

2300 Wastenaw Avenue

Ann Arbor, MI 48104

Telephone: (313) 668-2256

National Environmental Satellite, Data, and Information Service

Washington, DC 20233

Telephone: (301) 763-7190 
The National Environmental Satellite, Data, and Information Service includes the following data centers:

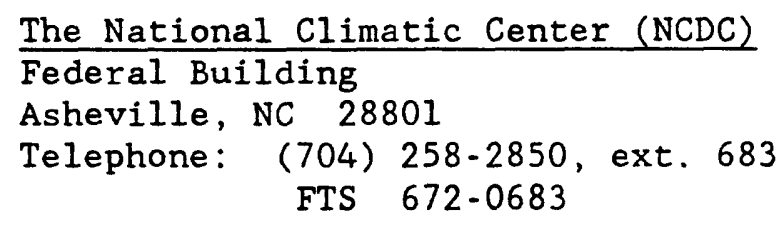

The National Oceanographic Data Center (NODC) Ocean Pollution Data and Information Network University Bldg, Room 415 Code: E/OC24 1825 Connecticut Ave, N.W. Washington, DC 20235

Telephone: (202) 673-5539

FTS $673-5539$

\section{Climate Applications Branch}

NOAA/NESDIS/SAL/ORA

Federal Building

200 East Cherry Street

Columbia, MO 65201

Telephone: (314) 875-5263

National Ground Water Information Center

National Water Well Association

6375 Riverside Drive

Dublin, $\mathrm{OH} 43917$

Designated Representative:

Kevin B. McCray, Director

Telephone: (614) 761-1711

National Park Service

Everglades National Park and

Fort Jefferson National Monument

P.O. Box 279

Homestead, FL 33030

Water Resources Division

$301 \mathrm{~S}$. Howes

Room 343

Fort Collins, CO 80521

Designated Representatives:

David A. Sikkema

Telephone: (305) 245-5266

FTS $350-4653$

Dave Ryn

Telephone: (303) 221-5341 


\section{Natural Science Systems}

1411 Hillcrest

Livonia, MI 48154

Designated Representative:

James Kubus

Telephone: (313) 525-1670

Nebraska Natural Resources Commission

301 Centennial Mall South, 4th Floor

P.O. Box 94876

Lincoln, NE 68509

Designated Representative:

Mahendra Bansal, Ph.D., P.E.

Head, Data Bank Section

State of Nebraska

Telephone: (402) 471-2081

Nevada Department of Conservation and Natural Resources

Division of Environmental Protection

201 South Fall Street

Room 221

Carson City, NV 89710

Designated Representative:

Ralph G. Capurro

Telephone: (702) 885-4670

New Castle County Water Resources Agency

2701 Capitol Trail

Newark, DE 19711

Designated Representatives:

Robert W. Finkle, Data Systems Manager

Bernard Dworsky, Administrator

Telephone: (302) 731-7670

New Jersey Geological Survey

P.O. Box $\mathrm{CN}-029$

Trenton, NJ 08625

Designated Representatives:

Haig Kasabach, State Geologist

Telephone: (609) 292-1185

Gail Carter

Bureau of Ground Water Resources

Evaluation (BGWRE)

Telephone: (609) 984-6587 
New Mexico Environmental Improvement Division

Surface Water Quality Bureau

1190 St. Francis Drive

Runnels B1dg. N2109

Santa Fe, NM 87501

Designated Representative:

Don Ditmore, Programmer Analyst

Telephone: (505) 827-2823

New Mexico State Engineers Office

State Engineers office

Bataan Memorial Building

Santa $\mathrm{Fe}$, NM

Designated Representative:

Francis G. West

Telephone: (505) 827-6154

New York State Department of Environmental Conservation

50 Wolf Road

Room 328

Albany, NY 12233-3503

Designated Representative:

George Hansen, Chief, Quality Assessment Section

Telephone: (518) 457-8819

Noah Corporation

120 Calumet Court

Aiken, SC 29801

Designated Representative:

James B. Price

Telephone: (803) 642-2749

North American Hydro, Inc.

P.O. Box 167

Neshkoro, WI 54960

Designated Representative:

Loyal Gake

Telephone: (414) 293-4628 
NRCD/DEM

512 N. Salisbury Street

P.O. Box 27687

Raleigh, NC 27611

Designated Representative:

Elora M. Lee

Telephone: (919) 733-7015

North Dakota Geological Survey

600 E. Boulevard

Bismarck, ND 58505

Designated Representative:

Sidney B. Anderson

Telephone: (701) 224-4109

North Dakota State Water Commission

900 East Boulevard

Bismarck, ND 58505

Designated Representative:

David A. Sprynczynatyk, Director of Engineering

Telephone: (701) 224-2750

Northeast Engineering Associates, Inc.

2317 Black Rock Turnpike

Fairfield, CT 06430

Designated Representative:

Bronislaw Janicki

Telephone: (203) 372-6998

Northwest Florida Water Management District

Route 1, Box 3100

Havana, FL 32333

Designated Representative:

David L. Barton

Telephone: (904) 487-1770 
Northwest Hydraulic Consultants, Inc.

22017 70th Avenue South

Kent, WA 98032

Designated Representative

Evan E. Twombly

Telephone: (206) 872-0218

Nuclear Regulatory Commission (U.S.)

M. S. WFO $4 \mathrm{H} 3$

Washington, DC 20555

Designated Representative:

Dr. Donald L. Chevy, Jr., Section Leader

Geosciences and Systems Performances Branch

U.S. Nuclear Regulatory Commission, NMSS

Telephone: (202) 492-3461

OTT Engineering, Inc.

1412 140th Place, NE

Bellevue, WA 98007

Designated Representatives:

Dennis Dorrateague

Telephone: (206) 747-1126

(Alternate: Roger A. Clay)

Oak Ridge National Laboratory

P.O. Box 2008, Building 1505

Oak Ridge, TN 37831-6038

Designated Representative:

Carolyn T. Hunsaker

Telephone: (615) 574-7365

FTS 624-7365 
U.S. Department of the Interior

South Interior Building

1951 Constitution Avenue

Room 5101L

Washington, DC 20240

Designated Representatives:

Douglas Growitz

Telephone: (202) 343-1507

FTS 343-1507

Office of Surface Mining

Technical Assistance Division

Parkway Center, Building 10

Pittsburgh, PA 15220

Chief, Hydrology Branch

Telephone: (412) 937-2153

FTS 564-2153
Office of Surface Mining

Brook Towers

102015 th Street

Denver, CO 80202

Chief, Hydrology Branch

Telephone: (303) 844-2578

FTS 564-2578

\section{Ohio Environmental Protection Agency}

1030 King Avenue

Columbus, $\mathrm{OH} \quad 43212$

Designated Representative:

Dennis B. Mishne, Data Coordinator

Telephone: (614) 466-3700

Ohio River Valley Water Sanitation Commission

49 East Fourth Street

Suite 815

Cincinnati, OH 45202

Designated Representatives:

Richard L. Herd, Jr., Principal

Telephone: (513) 421-1151

(Alternates: Christian G. Norman and Jason P. Heath)

Oklahoma Climatological Survey

710 Asp Avenue

Suite No. 8

Norman, OK 73019

Designated Representative:

Dr. Kenneth C. Crawford, Director \& State Climatologist

Telephone: (405) 325-2541 
Oklahoma State Department of Health

P.O. Box 53551

Oklahoma City, OK 73152

Designated Representative:

Judith A. Duncan, Chief

State Environmental Laboratory

Telephone: (405) 271-5240

Oklahoma State University, Department of Geology

Stillwater, OK 74078

Designated Representative:

Prof. Arthur W. Hounslow

Telephone: (405) 744-5917

Oklahoma Water Resources Board

1000 N.E. 10th Street

P.0. Box 53585

Oklahoma City, OK 73152

Designated Representative:

Jan Hook

Telephone: (405) 271-2585

P. E. LaMoreaux and Associates

P. O. Box 2310

Tuscaloosa, AL 35403

Designated Representative:

James W. LaMoreaux

Telephone: (205) 752-5543

P. G. International, Inc.

P.O. Box 3298

Annapolis, MD 21403

Designated Representative:

Phyllis G. Thompson

Telephone: (301) 267-9548 
15375 SE 30th PL

Suite 250

Bellevue, WA 98007

Designated Representative:

Kerry W. Ritland

Telephone: (206) 643-9803

Parsons, Brinckerhoff, Quade and Douglas, Inc.

1510 Arden Way

Suite 301

Sacramento, CA 95815

Designated Representative:

Tad Widby

Telephone: (916) 925-5535

Pat Harrison Waterway District

P.0. Drawer 1509

Hattiesburg, MS 39401

Designated Representative:

William H. Reid, Dist Engineer

Telephone: (601) 264-5951

Pendleton, Oregon, Public Works

P.O. Box 190

Pendleton, OR 97801

Designated Representative:

Gerald L. Odman, Public Works Director

Telephone: (503) 276-1811

Pennsylvania Department of Environmental Resources

Commonwealth of Pennsylvania

P.0. Box 1467

Harrisburg, PA 17105-1467

Designated Representative:

William Gast, Chief, State Water Plan Division

Telephone: (717) 541-7805 
Pollution Engineering

1935 Shermer Road

Northbrook, IL 60062

Designated Representative:

Richard A. Young, Editor

Telephone: (312) 498-9840

Post, Buckley, Schuh \& Jernigan, Inc.

8600 Northwest 36 Street

Miami, FL 33166-6622

Designated Representatives:

William Pitt, P.E., P.H.

Telephone: (305) 592-7275

RMC-Environmental Services

P.O. Box 10

1921 River Road

Drumore, PA 17518

Designated Representative:

Susan A. Haney

Telephone: (717) 548-2121

R. E. Wright Associates, Inc.

3240 Schoolhouse Road

Middletown, PA 17057

Designated Representative:

Richard E. Wright, President

Telephone: (717) 944-5501

R. W. Beck and Associates

Fourth and Blanchard Building

Suite 600

2101 Fourth Avenue

Seattle, WA 98121-2375

Designated Representative:

A. Richard Griffith

Telephone: (206) 441-7500
300 Atlanta Technology Center 1575 Northside Drive

Atlanta, GA 30318-4203

John C. Bausano, P.E.

Telephone: (404) 351-5608 
$\underline{R} \& \mathrm{M}$ Consultants, Inc.

5024 Cordova Street

Anchorage, AK 99503

Designated Representative:

Jeffrey H. Coffin

Telephone: (907) 561-1733

$\underline{\text { Radian Environmental Management }}$

Radian Corporation

8501 Mopac Boulevard

P.O. Box 201088

Austin, TX 78720-1088

Designated Representatives:

James L. Machin, Senior Engineer

Telephone: (512) 454-4797

Randolph \& Associates, Inc.

8901 North Industrial Road

Peoria, IL 61615

Designated Representative:

Erik I. Sloneker, Hydrogeologist

Telephone: (309) 692-4422

Raymond Vail \& Associates

1410 Ethan Way

Sacramento, CA 95825

Designated Representative:

Rex B. Clement

Telephone: (916) 929-3323

Reedy Creek Improvement District

P.0. Box 10170

Lake Buena Vista, FL 32830-0170

Designated Representative:

Gary Gornto, Manager, Environmental Department

Telephone: (407) 824-7311
5101 West Beloit Road

Milwaukee, WI 53214

Charles S. Applegate, Lab Manager Telephone: (414) 643-2768 


\section{Research Triangle Institute}

3040 Cornwallis Road

P.O. Box 12194

Research Triangle Park, NC 27709

Designated Representative:

Richard Pratt

Telephone: (919) 541-7137

Rio Blanco Oil Shale Company

4380 South Syracuse Street

Suite 500

Denver, CO 80237

Designated Representative:

Vern W. Butler

Telephone: (303) 889-0155

Roald Haestad, Inc.

37 Brookside Road

Waterbury, CT 06708

Designated Representative:

Salvatore A. Longo

Telephone: (203) 753-9800

Rocky Mountain Hydro, Inc.

4065 South Roslyn Street

Denver, CO 80237

Designated Representative:

Tom J. Rawlings

Telephone: (303) 770-9191

Roy F. Weston, Inc.

Weston Environmental Consultants--Designers Weston Way

West Chester, PA 19380

Designated Representative:

Ron Ragan

Telephone: (215) 692-3030
5599 San Felipe

Suite 700

Houston, TX 77056

David E. Mohr

Telephone: (713) 621-1620 
11260 Roger Bacon Drive

Reston, VA 22090

Designated Representative:

Jeffrey L. Briggs

Telephone: (703) 471-6150

(Alternate: Robert W. Luce)

SS Papadopulos \& Associates

12250 Rockville Pike

Suite 290

Rockville, MD 20852

Designated Representatives:

Steven Larson

Charlie Andrews

Telephone: (301) 468-5760

\section{Salt River Project}

Box 52025

Phoenix, AZ 85072-2025

Designated Representative:

James J. Krause, Water Resources Analyst

Telephone: (602) 236-2589

Santa Clara (City of) Water Department

1500 Warburton Avenue

Santa Clara, CA 95050

Designated Representative:

Richard H. Hathorn

Telephone: (408) 984-3183

Santa Clara Valley Water District

5750 Almaden Expressway

San Jose, CA 95118

Designated Representative:

Ms. J. Maher

Operations and Maintenance Division

Telephone: (408) 265-2600 
Sargent \& Lundy Engineers

55 East Monroe Street, 27th Floor Chicago, IL 60603

Designated Representative:

Walter C. Hannenberg

Telephone: (312) 269-3643

Scripps Institute of Oceanography

Mail Code S-002

University of California

LaJolla, CA 92093

Designated Representative:

George C. Anderson

Marine Physical Lab

Telephone: (619) 534-2582

Sierra/Misco, Inc.

1900 Point West Way

Suite 208

Sacramento, CA 95815-4703

Designated Representative:

David Leader

Telephone: (916) 929-8861

Simons, Li and Associates, Inc.

P.O. Box 1816

Fort Collins, CO 80522

Designated Representative:

Gregory J. Koch

Telephone: (303) 223-4100

Sithe Energies USA, Inc.

135 East 57 th Street

New York, NY 10022

Designated Representative:

Joern Seigies

Telephone: (212) 755-7600 
Soil Conservation Service (U.S.)

U.S. Department of Agriculture

Independence Ave. between 12 th and 14th Streets, S.W.

Washington, DC 20250

Telephone: (202) 447-4543

Designated Representatives:

Norman Miller

Ronald A. Jones

Engineering Division

10000 Aerospace Road

Lanham, MD 20801

Telephone: (301) 436-7383

FTS $436-7383$

201 East Indianola Avenue

Suite 200

Phoenix, AZ 85012

Telephone: (602) 241-2242

FTS 261-2242

Russe11 W. Craddock

Stephen R. Christensen

310 New Bern Avenue

333 Waller Avenue

5 th Floor, Federal Building

Raleigh, NC 27601

Telephone: (919) 790-2898

Lexington, KY 40504

Telephone: (606) 233-2502

Snow Survey and Water Supply

Forecasting, SCS-USDA

David E. Johnson, Program Manager

511 NW Broadway, Room 248

Portland, OR 97209-3489

Telephone: (FTS) 423-2843

Vermont SCS District office

Robin 0. Allen

69 Union Street

Winooski, VT 05404

Telephone: (802) 951-6795

Sonoma County Water Agency

P.O. Box 11628

Santa Rosa, CA 95406

Designated Representative:

Walt Koons

Telephone: (707) 526-5370

South Florida Environmental Research Foundation

4660 122nd Drive, North

Royal Palm Beach, FL 33411

Designated Representative:

Sally Black

Telephone: (305) 793-0582 
Southern California Edison Company

P.O. Box 800

2244 Walnut Grove Avenue

Rosemead, CA 91770

Designated Representative:

Jack Y. Kawashima

Telephone: (818) 302-9725

Southern Company Services, Inc.

Geotechnical/Hydro

P.O. Box 2625

Building 44, Room 540

Birmingham, AL 35202

Designated Representative:

Shah W. Khan

Telephone: (205) 870-6219

Southwest Florida Water Management District

2379 Broad Street

Brooksville, FL 33512-9712

Designated Representative:

Terry Hidgon

Telephone: (904) 796-7211

Southwest Georgia Regional Development Center

P.O. Box 346, City Hall

Camilla, GA 31730

Designated Representative:

Carroll C. Underwood, Executive Director

Telephone: (912) 336-5616

State of South Carolina, Water Resources Commission

1201 Main Street

Suite 212

Columbia, SC 29201

Designated Representative:

Teresa Greaney

Telephone: (803) 737-0800 
Stetson Engineers, Inc.

2171 East Francisco Blvd.

San Rafael, CA 94901

Designated Representative:

Richard J. Paulson

Telephone: (415) 457-0701

Susquehanna River Basin Commission

1721 North Front Street

Harrisburg, PA 17102

Designated Representative:

Marshall S. Goulding, Chief Engineer

Telephone: (717) 238-0425

Suwannee River Water Management District

Route 3, Box 64

Live Oak, FL 32060

Designated Representative:

Donad Monroe

Telephone: (904) 362-1001

Systems for Water Information Management

Minnesota State Planning Agency

Land Management Information Center

300 Centennial Building

658 Cedar Street

St. Paul, MN 55155

Designated Respresentative:

Susanne Maeder

Telephone: (612) 297-4986

Tampa Bay Engineering, Inc.

2240 Belleair Road

Suite 200

Clearwater, FL 34624

Designated Representative:

E. Peter Nikolov, P.E.

Telephone: (813) 531-3505 
Tenera, L. P.

308 N. Peters Road

Suite 280

Knoxville, TN 37922

Designated Representative:

Michael C. Meyers

Telephone: (615) 531-0806

TenEch Environmental Engineers, Inc.

515 Park Avenue

Louisville, KY 40208

Designated Representative:

Robert Hawkins

Telephone: (502) 636-3565

Other TenEch Offices

744 West Washington Street

9635 Saric Court

South Bend, IN 46601

Highland, IN 46322

Telephone: (219) 234-1166

Telephone: (219) 924-1018

Tennessee Department of Health and Environment

Bureau of Environment

Division of Water Supply

T.E.R.R.A. Building

1509 th Avenue North

Nashville, TN 37219-5404

Designated Representative:

A. Janine Morris

Telephone: (615) 741-6623

Tennessee Valley Authority (U.S.)

303 Evans Building

Knoxville, TN 37902

Designated Representative:

Robert C. Beebe, Data Systems

Telephone: (615) 632-4203 
Tetra Tech, Inc.

348 W. Hospitality Lane

San Bernardino, CA 92408

Designated Representatives:

David Cargo, Senior Geohydrologist

Telephone: (714) 381-1674

Texas Natural Resources Information System

P.O. Box 13231, Capitol Station

Austin, TX 78711

Designated Representative:

Dr. Tommy Knowles, Chairman

TNRIS Task Force

Telephone: (512) 463-8407

The preceding organization represents the following independent organizations:

Texas Water Development Board

P.0. Box 13087

Capitol Station

Austin, TX 78711

Designated Representative:

Dr. Tommy Knowles

Telephone: (512) 463-8407

Texas General Land Office

1700 North Congress Avenue

Stephen B. Austin Building

Austin, TX 78701

Designated Representative:

Sally Davenport

Telephone: (512) 463-5059

Texas Air Control Board

6330 Highway 290 East

Austin, TX 78723

Designated Representative:

Dr. Tom Porter

Telephone: (512) 451-5711, ext. 225 
Texas Forest Service

Texas A\&M University

College Station, TX 77843

Designated Representative:

Roger Lord

Telephone: (409) 845-2641

Texas Economic Development Commission

P.0. Box 12728

Capitol Station

Austin, TX 78711

Designated Representative:

Charles Newell

Telephone: (512) 472-5059 ext. 626

Texas Department of Health

1100 West 49 th Street

Austin, TX 78756-3199

Designated Representative:

Glendon Eppler

Telephone: (512) 458-7271

Bureau of Economic Geology (University of Texas at Austin)

Box $X$, University Station

Austin, TX 78713

Designated Representative:

Dr. E. G. Wermund, Jr.

Telephone: (512) 471-1534 ext. 128

Railroad Commission of Texas

P.0. Drawer 12967

Capitol Station

Austin, TX 78711-2967

Designated Representative:

Jimmy Walker

Telephone: (512) 463-6838

Texas Department of Agriculture

P.0. Box 70

Austin, TX 78767

Designated Representative:

Robert King

Telephone: (512) 463-7504 
Texas Historical Commission

Office of the State Archeologist

P.O. Box 12276

Capitol Station

Austin, TX 78711

Designated Representative:

Robert J. Mallouf

(512) 463-6090

Texas State Department of Highways and Public Transportation Highway Building

Brazos and East 11th Streets

Austin, TX 78701

Designated Representative:

Roger Merrell

Telephone: (512) 465-7337

Texas State Soil and Water Conservation Board

P.0. Box 658

Temple, TX 76503

Designated Representative:

Mel Davis

Telephone: (817) 773-2250

Texas Water Commission

P.0. Box 13087

Capitol Station

Austin, TX 78711

Designated Representative:

Bill Klemt

Telephone: (512) 371-6320

The Chester Engineers, Inc.

845 Fourth Avenue

Corapolis, PA 15108

Designated Representative:

Dr. Uzair M. Shamsi

Telephone: (412) 269-5907 
The Dow Chemical Company

P.O. Box 1706

9001 Building

Midland, Michigan 48641

Designated Representative:

Dr. James Knuteson

Telephone: (517) 636-9242

The Environ Corporation

210 Carnegie Center

Suite 201

Princeton, NJ 08540

Designated Representative:

Robert B. Gierzoff

Telephone: (609) 452-9000

Tudor Engineering Company

Consulting Engineers \& Planners

301 Mission Street

San Francisco, CA 94105

Designated Representative:

S. T. Su

Telephone: (415) 543-9820

Uintex Corporation

4568 Highland Drive, \#240

Salt Lake City, UT 84117

Designated Representative:

Dr. Anching Lin

Telephone: (801) 272-5239

University of Arizona, College of Business and Public Administration

Department of Management Information Systems

Tucson, AZ 85721

Designated Representative:

Dr. J. F. Nunamaker

Professor of Management Information Systems

College of Business and Public Administration

Telephone: (602) 621-4475 
College Eight

Santa Cruz, CA 95064

Designated Representative:

Dr. Robert R. Curry

Telephone: (408) 429-2900

University of Maryland, Department of Civil Engineering

Remote Sensing Systems Lab

College Park, MD 20742

Designated Representative:

Dr. Robert M. Ragan

Telephone: (301) 454-3107

Updata Publications, Inc.

1746 Westwood Boulevard

Los Angeles, CA 90024

Designated Representative:

Herbert Sclar

Telephone: (213) 474-5900

Upper Colorado River Commission

355 South Fourth East Street

Salt Lake City, UT 84111

Designated Representative:

Gerald R. Zimmerman, Executive Director

Telephone: (801) 531-1150

Utah Department of Natural Resources, Division of Water Rights

1636 West North Temple

Salt Lake City, UT 84116

Designated Representative:

Craig D. Morgan

Telephone: (801) 538-7392

FTS $8-(801)-533-6071$ 
Building 10 North

103 S. Main Street

Waterbury, VT 05676

Designated Representative:

David Butterfield

Telephone: (802) 244-5638

Versar, Inc.

6850 Versar Center

Springfield, VA 22151

Designated Representative:

Miquel J. Trespalacios

Telephone: (703) 750-3000

Virginia Beach Department of Public Utilities

Water Resources Division

Municipal Center

Virginia Beach, VA 23456-9002

Designated Representative:

Rita Sweet

Telephone: (804) 427-8035

WBLA, Inc.

1002 Walnut

Suite 200

Boulder, CO 80302

Designated Representative:

Benjamin L. Harding

Telephone: (303) 443-7839

W. W. Wheeler and Associates, Inc.

3700 South Inca Street

Englewood, CO 80110

Designated Representative:

James A. Ferentchak, P.E.

Telephone: (303) 761-4130 
Wagner, Heindel, and Noyes, Inc.

P.0. Box 1629

Burlington, Vermont 05402-1629

Designated Representative:

Robin Russo

Telephone: (802) 658-0820

Wapora, Inc.

7926 Jones Branch Drive

Suite 1100

McLean, VA 22102

Designated Representative:

Carol Vassallo

Telephone: (703) 893-3904

Warzyn Engineering, Inc.

715 Florida Avenue

Minneapolis, MN 55426

Designated Representative:

Craig Johnson

Telephone: (612) 593-5650

Washington Department of Ecology

Olympia, WA 98504

Telephone: (206) 459-6000

Designated Representative:

Art Larson

Department of Ecology

Mail Stop PV-11

Telephone: (206) 459-6082

Washington Department of Fisheries, Habitat Management Division

115 General Administration Building

Olympia, WA 98504

Designated Representative:

Ken Bates

Telephone: (206) 753-6600 
UOG Station

Mangilao, Guam 96913

Designated Representative:

Dr. Shahram Khosrowpanah, Acting Director

Telephone: (671) 734-3132

Fax (671) $734-3118$

Water Engineering and Technology, Inc.

419 Canyon Avenue

Suite 225

P.O. Box 1946

Fort Collins, CO 80522

Designated Representative:

Chester C. Watson

Telephone: (303) 482-8201

Water Research Center, Washington State University

State of Washington Water Research Center

Washington State University

Pullman, WA 99164-3002

Designated Representative:

William Funk, Director

Telephone: (509) 335-5531

Water Resources Center, Nevada University System

Desert Research Institute

Suite 1

2505 Chandler Avenue

Las Vegas, NV 89120

Designated Representative:

Karl Pohlmann

Telephone: (702) 798-8882

Water Resources Center, Ohio State University

1791 Neil Avenue

Columbus, $\mathrm{OH} \quad 43210$

Designated Representative:

Dr. Robert C. Stiefel, Director

Telephone: (614) 292-2334 
Water Resources Center, Rhode Island University

204 B1iss Hall

Kingston, RI 02881

Designated Representative:

Dr. Calvin P. C. Poon, Director

Telephone: (401) 792-2297

Water Resources Center, University of Delaware

210 Hullihen Hall

Newark, DE 19716

Designated Representative:

Dr. Robert D. Varrin, Director

Telephone: (302) 451-2191

Water Resources Center, University of Illinois at Urbana-Champaign

205 North Mathews

Urbana, IL 61801

Designated Representative:

Dr. Glenn E. Stout, Director

Telephone: (217) 333-0536

FTS 8-(217)-333-0536

Water Resources Center, University of Wisconsin

1975 Willow Drive

Madison, WI 53706

Designated Representative:

Dr. Gordon Chesters

Telephone: (608) 262-3838

Water Resources Research Center, Arizona University

Geology Building

Room 318

Tucson, AZ 85721

Designated Representative:

Marvin Waterstone

Telephone: (602) 621-7607 
Van Ness Campus

4200 Connecticut Avenue, N.W.

Washington, DC 20008

Designated Representative:

Dr. Mamadou H. Watt, Director

Telephone: (202) 673-3442

Water Resources Research Center, Massachusetts University

Blaisdell House

Amherst, MA 01003

Designated Representative:

Paul J. Godfrey, Director

Telephone: (413) 545-2842

Water Resources Research Center, University of Minnesota

866 Biological Sciences Center

University of Minnesota

St. Paul, MN 55108

Designated Representative:

Dr. Patrick L. Brezonik, Director

Telephone: (612) 624-3738

Water Resources Research Center, University of Missouri

0056 Engineering Complex

Columbia, MO 65211

Designated Representative:

Dr. Thomas E. Clevenger, Director

Telephone: (314) 882-3132

Water Resources Research Center, University of New Hampshire

218 Science/Engineering Researc.. Building

Durham, NH 03824

Designated Representative:

Dr. Thomas P. Ballestero, Director

Telephone: (603) 862-2144 
Water Resources Research Center, Virginia Polytechnic Institute and State University

617 North Main Street

Blacksburg, VA 24060-3397

Designated Representative:

T. W. Johnson, Assistant to the Director

Virginia Water Resources Research Center

Telephone: (703) 231-8037

FTS $8-961-5624$

Water Resources Research Institute, North Dakota State University

Fargo, ND 58105

Designated Representative:

Dr. R. Craig Schnell

Telephone: (701) 237-7033

Water Resources Research Institute, South Dakota State University

Brookings, SD 57007

Designated Representative:

Alan R. Bender, Acting Director

Telephone: (605) 688-4910

Water Resources Research Institute, University of Idaho

Agricultural Engineering Department

University of Idaho

Moscow, ID 83843

Designated Representative:

Dr. Myron Molnau

Telephone: (208) 885-6182

Water Resources Research Institute, University of Kentucky

219 Anderson $\mathrm{Hall}$

Lexington, KY 40506-0046

Designated Representative:

Dr. Ralph R. Huffsey

Telephone: (606) 257-1832 
Water Resources Research Institute, University of Puerto Rico

University of Puerto Rico, - R.U.M

P.0. Box 5000

Mayaguez, PR 00709-5000

Designated Representative:

Prof. Luis A. del Valle, Director

Telephone: (809) 832-4040, ext. 2048

Water Resources Research Institute, University of North Carolina

Box 7912

North Carolina State University

Raleigh, NC 27695-7912

Designated Representative:

Dr. David H. Moreau, Director

Telephone: (919) 737-2815

WaterTest Corporation

P.0. Box 6360

Manchester, NH 03108-6360

Designated Representative:

Paul M. Porter

Telephone: (603) 623-7400

Western Engineers, Inc.

2150 Highways 6 and 50

Grand Junction, CO 81505

Designated Representative:

John M. Currier

Telephone: (203) 242-5202

Western Hydrologic Systems

3037 Grass Valley Highway 8201

Auburn, CA 95603

Designated Representative:

Patrick B. Cawood

Telephone: (916) 885-2480 
William $\mathrm{T}$. Lorenz and Company

Management Consultants

85 Warren Street

Concord, NH 03301

Designated Representative:

William T. Lorenz, President

Telephone: (603) 228-3373

Winter Haven Lake Region Boat Course District

804 Avenue X, S.W.

Winter Haven, FL 33880

Designated Representative:

Roger Griffiths

Telephone: (813) 293-1441

Wisconsin Geological and Natural History Survey

3817 Mineral Point Road

Madison, WI 53705

Designated Representative:

Kathleen M. Massie-Ferch

Telephone: (608) 262-9468

Woodward-Clyde Consultants

Suite 1000

4582 South Ulster Street Parkway

Denver, CO 80237

Designated Representative:

Janna Jantz

Telephone: (303) 694-2770

Wright-Pierce Engineers

99 Main Street

Topsham, ME 04086

Designated Representative:

Gregg Carrington

Telephone: (207) 725-9721 
Wright Water Engineers, Inc.

2490 West 26 th Avenue

Suite 100-A

Denver, CO 80211

Designated Representative:

Dwight $W$. Kimsey

Telephone: (303) 480-1700

Wyoming Department of Environmental Quality

Hathaway Building

2300 Capitol Avenue

Cheyenne, WY 82002

Designated Representative:

Edward Mock, Ph.D.

Telephone: (307) 777-7317

Wyoming Water Research Center

University of Wyoming

Box 3067, University Station

Laramie, WY 82071

Designated Representative:

Barry Lawrence

Telephone: (307) 766-2143

Yonts and Associates

Water Resources, Community Planning and

Engineering Consultants

508 North Glen Drive

Raleigh, NC 27609

Designated Representative:

Woodrow L. Yonts

Telephone: (919) 787-7378

Young Environmental Services, Inc.

1253 Roosevelt Avenue

Glenview, IL 60025

Designated Representative:

Carol S. Young

Telephone: (312) 724-6631 
Aqua Mex, S.A.

Apdo Postal 2581

Monterrey, N.L., 64000

Mexico

Designated Representative:

Enrique A. Gonzalez Guzman, P.E.

General Manager

Telephone: (52-83) 33-40-15;33-40-17; 33-31-43

Companhia Auxiliar de Empresas Electricas Brasileiras (CAEEB)

SAS ED. ASCB 11th Andar

70070 Brasilia - DF Brasil

Telephone: (061) 223-8544

Designated Representative:

Dr. Riccardo Jose Cioglia

Chief, Department of Data Processing

National Institute of Hydrology

University of Roorkee Campus

Roorkee 247667 (U.P.) India

Designated Representative:

Dr. Satish Chandra, Director

Telephone: +91-1332-2106

Telex:0597-205 NIH IN

Nigerian Water Data Bank

Faculty of Engineering

University of Lagos

Lagos, Nigeria

Designated Representative:

Professor Oye Ibidapo-Obe

Deputy Project Leader 
South African Water Information Centre, CSIR

P.O. Box 395

Pretoria 0001

South Africa

Designated Representative:

AG James, Project Manager

Telephone: $2712841-3083$

Water Resources Document Reference Centere

Inland Waters/Lands Directorate

Environment Canada

ottawa Ontario

Canada KIA $\mathrm{OH} 3$

Designated Representative:

Ronald Gauthier

Water Management Systems Division

Water Planning and Management Branch

Telephone: (819) 953-1531

Water Resources Planning Commission

Hydrology Division

Ministry of Economic Affairs

12-F, 41-3, Sec 3, Hsin-I Road

Taipei, Taiwan

Republic of China

Designated Representative:

Alan Shaohsing King

Chief, Surface Water Section

Telephone: (02) 754-2080 ext. 810 


\author{
APPENDIX \\ MEMORANDUM OF UNDERSTANDING \\ BETWEEN \\ $* * * * * * * * * * * * * * * * * * * * * * * * * * * * * *$ \\ AND THE \\ UNITED STATES GEOLOGICAL SURVEY \\ PERTAINING TO THE NATIONAL WATER DATA EXCHANGE
}

The National Water Data Exchange (NAWDEX) is comprised of water-oriented organizations working together to provide convenient access to water data. The NAWDEX mission is to identify sources of water data, to index data holdings of water-oriented organizations, and to provide the linkage between those who acquire and those who use water data.

This memorandum recognizes $* * * * * * * * * * * * * * * * * * * * * * *$, as a participating member of the National Water Data Exchange (NAWDEX). This membership will continue in effect until terminated by mutual agreement or by either agency providing 60 days written notice to the other agency.

The U.S. Geological Survey (USGS), through its NAWDEX Program Office, will provide the central management of NAWDEX, and will serve as a coordinating facility for all NAWDEX facilities.

The NAWDEX Program Office will be responsible for:

- Establishing response and referral mechanisms for handling requests for water data in the files of NAWDEX members.

- Establishing and maintaining a Master Water Data Index of data holdings of the NAWDEX members and making the index available to all.

- Establishing and maintaining a Water Data Sources Directory and making this directory available to all.

- Establishing a nationwide network of NAWDEX Assistance Centers that will provide data search assistance to requesters and aid them in gaining access to water data held by NAWDEX members.

$* * * * * * * * * * * * * * * * * * * * * * * * * * * * * * * * * *$, will be responsible for:

- Taking an active role in the formulation of NAWDEX policies, procedures, and standards and implementing them within its organization to the extent practicable.

- Participating in the development of standard techniques and methodologies for handling of water data and using them within its organization to the extent practicable. 
- Providing information on internally held water data for inclusion in the Master Water Data Index and, as requested, providing current information to update the Master Water Data Index to reflect additions, changes, and corrections to the index.

- Providing data from its internal holdings either in response to a referral from the NAWDEX Program Office or a NAWDEX Assistance Center, or in response to a direct request for water data.

- Designating a representative of its organization to function as the primary contact for all NAWDEX matters.

It is mutually understood that membership in NAWDEX is voluntary and that all members will participate on an equal basis, and consent to be listed as a source of water data in the Water Data Sources Directory. There will be an open exchange of information among NAWDEX members and every effort will be made to provide water data to the user community in a timely and equitable manner.

Dr. James S. Burton

Program Manager

National Water Data Exchange

Signature, Title

Signature, Title

Date

Date 